\title{
Strichartz Estimate and Nonlinear Klein-Gordon Equation on Nontrapping Scattering Space
}

\author{
Junyong Zhang ${ }^{1,2}$. Jiqiang Zheng ${ }^{3,4}$
}

Received: 14 May 2018 / Published online: 4 October 2018

(c) The Author(s) 2018

\begin{abstract}
We study the nonlinear Klein-Gordon equation on a product space $M=\mathbb{R} \times X$ with metric $\tilde{g}=d t^{2}-g$ where $g$ is the scattering metric on $X$. We establish the global-intime Strichartz estimate for Klein-Gordon equation without loss of derivative by using the microlocalized spectral measure of Laplacian on scattering manifold showed in Hassell and Zhang (Anal PDE 9:151-192, 2016) and a Littlewood-Paley squarefunction estimate proved in Zhang (Adv Math 271: 91-111, 2015). We prove the global existence and scattering for a family of nonlinear Klein-Gordon equations for small initial data with minimum regularity on this setting.
\end{abstract}

Keywords Strichartz estimate $\cdot$ Scattering manifold $\cdot$ Spectral measure $\cdot$ Global existence $\cdot$ Scattering theory

Mathematics Subject Classification 35Q40 · 35S30 · 47J35

\section{Introduction and Statement of Main Results}

In this paper we consider the evolution of a semilinear Klein-Gordon equations with power-type nonlinearities on a nontrapping scattering manifold. More specifically, we

Junyong Zhang

zhang_junyong@bit.edu.cn; ZhangJ107@cardiff.ac.uk

Jiqiang Zheng

zhengjiqiang@gmail.com

1 Department of Mathematics, Beijing Institute of Technology, Beijing 100081, China

2 School of Mathematics, Cardiff University, Cardiff CF24 4AG, UK

3 Institute of Applied Physics and Computational Mathematics, Beijing 100088, China

4 Université Nice Sophia-Antipolis, 06108 Nice Cedex 02, France 
consider the following family of nonlinear Klein-Gordon equation

$$
\square \tilde{g} u+m^{2} u=F(u, D u), \quad(t, z) \in \mathbb{R} \times X, \quad u(0)=u_{0}(z), \partial_{t} u(0)=u_{1}(z) .
$$

Here $\square_{\tilde{g}}=\partial_{t}^{2}-\Delta_{g}$ denotes the d'Alembertian in the metric $\tilde{g}=d t^{2}-g$ and $\Delta_{g}$ is the Laplacian on the manifold $X$ with scattering metric $g$ introduced by Melrose [34]. We focus on the questions of the minimum regularity for which local well-posedness and small nonlinear scattering hold true. One of the motivations for this study is that in low-dimensional case, we can achieve the level of regularity corresponding to a conserved quantity (e.g., the energy) and thus get global existence for large initial data.

On more general class of physical manifolds, Hintz and Vasy [15-17,25] studied the semilinear and quasilinear wave and Klein-Gordon equation on the physically cosmological spacetimes as solutions to Einstein's field equations. In particular they gave a detailed analysis of the long-time behavior of linear and nonlinear waves on Kerr-de Sitter space and nontrapping Lorentzian scattering spaces for large regularity and small initial data. The recent development of [45] allowed them to set up the analysis of the associated linear problem in a framework of Fredholm problem, in which they used Melrose's philosophy $[33,34]$ of studying differential operators $P=$ $\square_{\tilde{g}}$ on a noncompact space $M$ by compactifying $M$ to a manifold $\bar{M}$ with boundary or even corners. The concrete choice of compactification is connected to the geometric structure of $\bar{M}$ near infinity. In our less complicated product setting, we use the same Melrose's idea with $P=\Delta_{g}$ on $X$ in the study of the spectral measure of the Laplacian $[15,26]$ and then analyze the propagator $e^{i t \sqrt{1-\Delta_{g}}}$; thus, we expect better result on the lowest regularity due to the establishment of the global-in-time Strichartz estimate.

In the simplest flat Euclidean space, there are a large number of literature to study the nonlinear Klein-Gordon equation. In the flat Euclidean space, where $X=\mathbb{R}^{n}$ and $g_{j k}=\delta_{j k}$, the dispersive properties of the Klein-Gordon and other dispersive equations have been proved to be powerful in the study of nonlinear problems. The Strichartz estimate for the solution of the homogenous and inhomogeneous KleinGordon equation in the form of space time integrability properties gives

$$
\begin{aligned}
& \|u(t, z)\|_{L_{t}^{q}\left(I ; L_{z}^{r}\left(\mathbb{R}^{n}\right)\right)}+\|u(t, z)\|_{C\left(I ; H^{s}\left(\mathbb{R}^{n}\right)\right)} \\
& \quad \lesssim\left\|u_{0}\right\|_{H^{s}\left(\mathbb{R}^{n}\right)}+\left\|u_{1}\right\|_{H^{s-1}\left(\mathbb{R}^{n}\right)}+\|F\|_{L_{t}^{\tilde{q}^{\prime}}\left(I ; L_{z}^{\tilde{r}^{\prime}}\left(\mathbb{R}^{n}\right)\right)},
\end{aligned}
$$

where the pairs $(q, r),(\tilde{q}, \tilde{r}) \in[2, \infty]^{2}$ satisfy the admissible condition for $0 \leqslant \theta \leqslant 1$

$$
\frac{2}{q}+\frac{n-1+\theta}{r} \leqslant \frac{n-1+\theta}{2}, \quad(q, r,(n-1+\theta) / 2) \neq(2, \infty, 1) .
$$

and the gap condition

$$
\frac{1}{q}+\frac{n+\theta}{r}=\frac{n+\theta}{2}-s=\frac{1}{\tilde{q}^{\prime}}+\frac{n+\theta}{\tilde{r}^{\prime}}-2
$$


We refer to Brenner [3], Ginibre-Velo [18], and Keel-Tao [29] for more details. In particular when $\theta=0$, these estimates corresponding to wave equation serve as a tool for existence results about the nonlinear wave equation. For example, LindbladSogge [32] answered the problem of finding minimal regularity conditions on the initial data ensuring local well-posedness for semilinear wave equations. Analogous results for the Klein-Gordon equation can be carried out even though the sharpness of well-posedness result is not known. There are too many references to cite all here, so we refer the reader to $[27,36]$ and the references therein.

In view of the rich Euclidean theory due to the Strichartz estimate, it is natural to consider the corresponding equations on more general manifolds. However it is difficult or impossible to establish the same Strichartz-type estimates as in Euclidean space on the large class of manifold due to the influence of qualitative geometric properties. On asymptotically de Sitter spaces Baskin [4-6] established a family of local (in time) weighted Strichartz estimates with derivative losses for the Klein-Gordon equation on asymptotically de Sitter spaces and provided a heuristic argument for the nonexistence of a global dispersive estimate on these spaces. The Strichartz estimates are local-intime or loss of derivatives on the compact manifold with or without boundary, see $[8,11,28,39,43]$ and references therein. On noncompact manifold with nontrapping condition, one can obtain global-in-time Strichartz estimates. For example, the global Strichartz estimates on a exterior manifold in $\mathbb{R}^{n}$ to a convex obstacle, for metrics $g$ which agrees with the Euclidean metric outside a compact set with nontrapping assumption, are obtained by Smith-Sogge [40] for odd dimension, and Burq [10] and Metcalfe [35] for even dimension. Blair-Ford-Marzuola [9] established global Strichartz estimates for the wave equation on flat cones $C\left(\mathbb{S}_{\rho}^{1}\right)$ by using the explicit representation of the fundamental solution. Anker-Pierfelice [2] study the problem on minimal regularity condition on the initial data ensuring well-posedness for wave and Klein-Gordon on hyperbolic space. On the nontrapping scattering manifold, the same setting considered here, Hassell, Tao, and Wunsch first established an $L_{t, z}^{4}$-Strichartz estimate for Schrödinger equation in [21] and then they [22] extended the estimate to full admissible local-in-time Strichartz estimate except endpoint $q=2$. More recently, Hassell-Zhang [26] improved the local-in-time one to global-in-time one and fixed the endpoint $q=2$ by analyzing the microlocalized spectral measure. Following this Zhang [47] extended the global-in-time result for the wave equation. Bouclet-Mizutani [7] generalized the Schrödinger result to the setting with mild trapping and with more general ends.

In this paper, we will establish the global-in-time Strichartz estimate for the Klein-Gordon and apply it to study the minimal regularity problem for nonlinear Klein-Gordon on the nontrapping scattering manifold (asymptotically conic manifold) which is the same as in $[22,26,47]$ including the asymptotically Euclidean space. The scattering manifold means that $X$ can be compactified to a manifold with boundary $\bar{X}$ such that $g$ becomes a scattering metric on $\bar{X}$; see more about this next section. For geometric reasons, we expect the same dispersive properties of Klein-Gordon as in the Euclidean setting. The key ingredient is to establish global-in-time Strichartz estimate for Klein-Gordon. It is known that Klein-Gordon behaves like Schrödinger at low frequency and wave equation at high frequency. Similar to the Euclidean space, we introduce a parameter $\theta$ for Klein-Gordon admissible pair which is wave admissi- 
ble at $\theta=0$ and Schrödinger pair at $\theta=1$. More precisely, we have the result about Strichartz estimates in the following.

Let $H^{s}(X)=\left(1-\Delta_{g}\right)^{-\frac{s}{2}} L^{2}(X)$ be the inhomogeneous Sobolev space over $X$. Throughout this paper, pairs of conjugate indices are written as $r, r^{\prime}$, where $\frac{1}{r}+\frac{1}{r^{\prime}}=1$ with $1 \leqslant r \leqslant \infty$.

Theorem 1.1 (Global-in-time Strichartz estimate) Let $(X, g)$ be nontrapping scattering manifold of dimension $n \geqslant 3$. Suppose that $u$ is the solution to the Cauchy problem

$$
\begin{cases}\partial_{t}^{2} u-\Delta_{g} u+u=F(t, z), & (t, z) \in I \times X \\ u(0)=u_{0}(z), & \partial_{t} u(0)=u_{1}(z),\end{cases}
$$

for some initial data $u_{0} \in H^{s}, u_{1} \in H^{s-1}$, and the time interval $I \subseteq \mathbb{R}$, then

$$
\begin{aligned}
& \|u(t, z)\|_{L_{t}^{q}\left(I ; L_{z}^{r}(X)\right)}+\|u(t, z)\|_{C\left(I ; H^{s}(X)\right)} \\
& \quad \lesssim\left\|u_{0}\right\|_{H^{s}(X)}+\left\|u_{1}\right\|_{H^{s-1}(X)}+\|F\|_{L_{t}^{\tilde{q}^{\prime}}\left(I ; L_{z}^{\tilde{r}^{\prime}}(X)\right)},
\end{aligned}
$$

where the pairs $(q, r),(\tilde{q}, \tilde{r}) \in[2, \infty]^{2}$ satisfy the KG-admissible condition with $0 \leqslant \theta \leqslant 1$

$$
\frac{2}{q}+\frac{n-1+\theta}{r} \leqslant \frac{n-1+\theta}{2}, \quad(q, r, n, \theta) \neq(2, \infty, 3,0) .
$$

and the gap condition

$$
\frac{1}{q}+\frac{n+\theta}{r}=\frac{n+\theta}{2}-s=\frac{1}{\tilde{q}^{\prime}}+\frac{n+\theta}{\tilde{r}^{\prime}}-2
$$

Remark 1.2 We remark that the estimates here are the same as the Strichartz estimates for Klein-Gordon on Euclidean space which are global-in-time and have no loss of derivatives.

We sketch the proof as follows. Similar to [26,47], our strategy is to use the abstract Strichartz estimate proved in Keel-Tao [29]. Thus, with $U(t)$ denoting the (abstract) propagator, we need to show uniform $L^{2} \rightarrow L^{2}$ estimate for $U(t)$, and $L^{1} \rightarrow L^{\infty}$ type dispersive estimate on the $U(t) U(s)^{*}$ with a bound of the form $O\left((1+|t-s|)^{-(n-1+\theta) / 2}\right)$ with $0 \leqslant \theta \leqslant 1$. In the flat Euclidean setting, the estimates are usually obtained by using stationary phase argument. One point is to write the propagator in the form of oscillatory integral. Since the Laplacian in our general setting is degenerate when it is close to the compactified boundary, the formulate turns out to be more complicated. On the other hand, the conjugate point occurring in this nonflat setting may lead to the failure of the dispersive estimate. For example, [24] showed that the Schrödinger propagator $e^{i t \Delta_{g}}$ failed to satisfy such a dispersive estimate at any pair of conjugate points $\left(z, z^{\prime}\right) \in X \times X$ (i.e., pairs $\left(z, z^{\prime}\right)$ where a geodesic emanating from $z$ has a conjugate point at $\left.z^{\prime}\right)$. Fortunately, we can localize the propagator to 
separate the conjugating points and write the propagator in a form of oscillatory integral by using a microlocalized spectral measure. The microlocalized spectral measure $Q_{j}(\lambda) d E \sqrt{-_{-}}(\lambda) Q_{j}(\lambda)^{*}$ constructed in [26] not only has a size estimate in decay rate but captures its oscillatory behavior, where $Q_{j}(\lambda)$ is a member of a partition of the identity operator in $L^{2}(X)$. In the stationary phase argument, the Klein-Gordon multiplier $e^{i t \sqrt{1+\lambda^{2}}}$ behaves like wave at high frequency and Schrödinger at low frequency. We establish the dispersive estimate with norm $O\left((1+|t-s|)^{-n / 2}\right)$ at low frequency and $O\left((1+|t-s|)^{-(n-1+\theta) / 2}\right)$ at high frequency. We finally show the Strichartz estimate from a frequency-localized Strichartz estimate by a square function estimate proved in [47]. The inhomogeneous Strichartz estimates follow from the homogeneous estimates and the Christ-Kiselev lemma.

Having the Strichartz estimate, we first consider the well-posedness and nonlinear scattering problem of the Cauchy problem on this setting

$$
\begin{cases}\partial_{t}^{2} u-\Delta_{g} u+u= \pm|u|^{p-1} u, & (t, z) \in \mathbb{R} \times X, \\ \left.u(t, z)\right|_{t=0}=u_{0}(z), & \left.\partial_{t} u(t, z)\right|_{t=0}=u_{1}(z) .\end{cases}
$$

In the case of flat Euclidean space, there are many results on the understanding of the global existence and scattering. We refer the readers to [32,42] and references therein. We here are mostly interested in the range of exponents $p \in\left[p_{\text {conf }}, 1+\frac{4}{n-2}\right]$ and the initial data are in $H^{s_{c}}(X) \times H^{s_{c}-1}(X)$, where $p_{\text {conf }}=1+\frac{4}{n}$ and $s_{c}=\frac{n}{2}-\frac{2}{p-1}$. The critical power $1+4 / n$, which is different from wave equation's $1+\frac{4}{n-1}$ in [47], is related to the dispersive estimate decay rate and it appears in the theorem because of the fact that NLKG is conformally invariant only if $F(u)=u^{1+4 / n}$; see [38]. The other power $1+4 /(n-2)$ is related to the energy-critical index.

The following result is about the well-posedness and nonlinear scattering with smallest regularity.

Theorem 1.3 Let $(X, g)$ be a nontrapping scattering manifold of dimension $n \geqslant 3$. Then if $\left(u_{0}, u_{1}\right) \in H^{s_{c}}(X) \times H^{s_{c}-1}(X)$ and $p \in\left[1+\frac{4}{n-1}, 1+\frac{4}{n-2}\right]$, there exist $T>0$ and a unique solution $u$ to (1.8) satisfying

$$
u \in C_{t}\left([0, T] ; H^{s_{c}}(X)\right) \cap L^{q_{0}}\left([0, T] ; L^{q_{0}}(X)\right),
$$

where $q_{0}=(p-1)(n+1) / 2$. In addition, if there is a small constant $\epsilon(p)$ such that

$$
\left\|u_{0}\right\|_{H^{s_{c}}}+\left\|u_{1}\right\|_{H^{s_{c}-1}}<\epsilon(p)
$$

then there is a unique global and scattering solution u to (1.8) satisfying

$$
u \in C_{t}\left(\mathbb{R} ; H^{s_{c}}(X)\right) \cap L^{q_{0}}\left(\mathbb{R} ; L^{q_{0}}(X)\right) .
$$

Furthermore if $\left(u_{0}, u_{1}\right) \in H^{1}(X) \times L^{2}(X)$ and $p \in\left[p_{\text {conf }}, 1+\frac{4}{n-2}\right)$, there exists a global solution to (1.8) with minus sign in the nonlinearity. 
Remark 1.4 On local well-posedness and small scattering with the minimal regularity result, we have to restrict ourself with $p \in\left[1+\frac{4}{n-1}, 1+\frac{4}{n-2}\right]$, that is, $s_{c} \geqslant 1 / 2$ and we can extend the similar result to $p \in\left[p_{\text {conf }}, 1+\frac{4}{n-1}\right]$ if $\left(u_{0}, u_{1}\right) \in H^{s}(X) \times H^{s-1}(X)$ with $s \geqslant 1 / 2$.

We next specially consider the well-posedness of the following Yang-Mills-type equations on this setting with dimension $n=3$.

$$
\begin{cases}\partial_{t}^{2} u-\Delta_{g} u+u=u D u+|u|^{2} u, & (t, z) \in \mathbb{R} \times X, \\ \left.u(t, z)\right|_{t=0}=u_{0}(z) \in H^{s}(X), & \left.\partial_{t} u(t, z)\right|_{t=0}=u_{1}(z) \in H^{s-1}(X) .\end{cases}
$$

The derivative $D u$ is measured relative to the metric structure; more precisely, $D$ is a first-order scattering differential operator. When dropping the linear term $u$, this equation has the same scaling as cubic NLW, but is more difficult technically because of the derivative term $u D u$. In the Euclidean space, this Yang-Mills-type wave equation was proved to be local well-posedness when $s>1$ in [37] and was showed to be ill-posedness when $s \leqslant 1$ in [31].

Theorem 1.5 Let $(X, g)$ be a nontrapping scattering manifold of dimension $n=3$ and let $0<\delta \ll 1$. Suppose $\left(u_{0}, u_{1}\right) \in H^{1+\delta}(X) \times H^{\delta}(X)$, then there exist $T>0$ and a unique solution $u$ to (1.8) satisfying

$$
u \in C_{t}\left([0, T] ; H^{1+\delta}(X)\right) \cap L^{2}\left([0, T] ; L^{\infty}(X)\right) .
$$

This paper is organized as follows. In Sect. 2 we review the background of scattering manifold, the results of the microlocalized spectral measure for the Laplacian, and the square function inequalities on this setting. Section 3 is devoted to the proofs of the microlocalized dispersive estimates and $L^{2}$-estimates. In Sect. 4 , we prove the homogeneous and inhomogeneous Strichartz estimates. Finally, we apply the Strichartz estimates to show Theorems 1.3 and 1.5.

\section{Some Analysis Tools on Scattering Manifold}

In this section, we briefly recall the key elements of the microlocalized spectral measure and a fundamental Littlewood-Paley squarefunction estimate. The first one was constructed by Hassell-Zhang [26] to capture both its size and the oscillatory behavior. The second one was proved in [47].

\subsection{Geometry Setting}

Let us recall the manifold with scattering metric introduced by Melrose [34]. There are many works to analyze the Laplacian operator on the scattering manifold, that is, asymptotically conic geometric setting; see [19,20,22,24,26]. Let $(X, g)$ be a complete noncompact Riemannian manifold of dimension $n \geqslant 2$ with one end, diffeomorphic 
to $(0, \infty) \times Y$ where $Y$ is a smooth compact connected manifold without boundary. Moreover, we assume $(X, g)$ is scattering manifold which means that $X$ allows a compactification $\bar{X}$ with boundary, with $\partial \bar{X}=Y$, such that the metric $g$ becomes an asymptotically conic metric on $\bar{X}$. In details, the metric $g$ in a collar neighborhood $[0, \epsilon)_{x} \times \partial \bar{X}$ near $Y$ takes the form of

$$
g=\frac{\mathrm{d} x^{2}}{x^{4}}+\frac{h(x)}{x^{2}}=\frac{\mathrm{d} x^{2}}{x^{4}}+\frac{\sum h_{j k}(x, y) d y^{j} d y^{k}}{x^{2}},
$$

where $x \in C^{\infty}(\bar{X})$ is a boundary defining function for $\partial \bar{X}$ and $h$ is a smooth family of metrics on $Y$. Here we use $y=\left(y_{1}, \ldots, y_{n-1}\right)$ for local coordinates on $Y=\partial M$, and the local coordinates $(x, y)$ on $\bar{X}$ near $\partial \bar{X}$. Away from $\partial \bar{X}$, we use $z=\left(z_{1}, \ldots, z_{n}\right)$ to denote the local coordinates. If $h_{j k}(x, y)=h_{j k}(y)$ is independent of $x$, we say $\bar{X}$ is perfectly conic near infinity. Moreover if every geodesic $z(s)$ in $\bar{X}$ reaches $Y$ as $s \rightarrow \pm \infty$, we say $\bar{X}$ is nontrapping. The function $r:=1 / x$ near $x=0$ can be thought of as a "radial" variable near infinity and $y$ can be regarded as the $n-1$ "angular" variables; the metric is asymptotic to the exact conic metric $\left((0, \infty)_{r} \times Y, d r^{2}+r^{2} h(0)\right)$ as $r \rightarrow \infty$. The Euclidean space $X=\mathbb{R}^{n}$ is an example of an asymptotically conic manifold with $Y=\mathbb{S}^{n-1}$ and the standard metric.

\subsection{The Laplacian on Scattering Manifold}

Our setting is on the scattering manifold, and we turn to the concepts of "scattering geometry." For a full discussion of scattering geometry, we refer the reader to Melrose [34]. The space of sc-vector fields is defined as $\left.\mathcal{V}_{\mathrm{sc}} \bar{X}\right)=x \mathcal{V}_{b}(\bar{X})$, where $\mathcal{V}_{b}(\bar{X})$ is the Lie algebra of all smooth vector fields on $\overline{X X}$ which are tangent to the boundary. The sc-vector field also forms a Lie algebra. These sc-vector fields can be realized as the sections of a vector bundle ${ }^{\mathrm{sc}} T \bar{X}$, called the sc-tangent bundle. That means $\mathcal{V}_{\mathrm{sc}}(\bar{X})=\mathcal{C}^{\infty}\left(\bar{X} ;{ }^{\mathrm{sc}} T \bar{X}\right)$, i.e., $\mathcal{V}_{\mathrm{sc}}(\bar{X})$ is a space of sections of ${ }^{\mathrm{sc}} T \bar{X}$ the sc-tangent bundle over $\bar{X}$. Using above notation in which $x$ is the boundary defining function of $\bar{X}$ and $y$ is the coordinate in $\partial \bar{X}$, we have

$$
\mathcal{V}_{\text {sc }}(\bar{X})= \begin{cases}\mathcal{V}, \text { i.e., all } \mathcal{C}^{\infty} \text {-vector fields, } & \text { in the interior } \bar{X} \\ \operatorname{span}\left\{x^{2} \partial_{x}, x \partial_{y_{1}} \ldots, x \partial_{y_{n-1}}\right\}, & \text { near the boundary } \partial \bar{X}\end{cases}
$$

We denote by $\operatorname{Diff}_{\mathrm{sc}}^{*}(\bar{X})$ the 'enveloping algebra' of $\mathcal{V}_{\mathrm{sc}}(\bar{X})$, meaning the ring of differential operator on $\mathcal{C}^{\infty}(\bar{X})$ generated by $\mathcal{V}_{\mathrm{sc}}(\bar{X})$ and $\mathcal{C}^{\infty}(\bar{X})$. In particular, near the boundary $\partial \bar{X}$, the $k$-order scattering differential operator is given by

$$
\operatorname{Diff}_{\mathrm{sc}}^{k}(\bar{X})=\left\{\mathcal{A}: \mathcal{A}=\sum_{j+|\alpha| \leqslant k} a_{j \alpha}(x, y)\left(x^{2} \partial_{x}\right)^{j}\left(x \partial_{y}\right)^{\alpha}, a_{j \alpha} \in C^{\infty}(\bar{X})\right\} .
$$


If $\alpha \in \mathbb{R}$, the $\alpha$ b-density bundle, denoted by ${ }^{\mathrm{sc}} \Omega^{\alpha}$, is defined by

$$
{ }^{\mathrm{sc}} \Omega^{\alpha} X=\bigcup_{p \in \bar{X}} \Omega^{\alpha}\left({ }^{\mathrm{sc}} T_{p} \bar{X}\right)
$$

In particular when $\alpha=1 / 2$, it is convenient to regard such operators as acting on schalf densities, that is, multiples of a half-density taking the form $\left|\frac{d x}{x^{2}} \frac{d y_{1}}{x} \ldots \frac{d y_{n-1}}{x}\right|^{1 / 2}$. Correspondingly, the Schwartz kernels of such operators can be written as a distribution tensored with a scattering half-density in each of the left and right variables.

Define ${ }^{\mathrm{sc}} T^{*} \bar{X}$, the scattering cotangent bundle over $\bar{X}$, to be the dual vector bundle to ${ }^{\mathrm{sc}} T \bar{X}$. Locally near the boundary, in the coordinate $(x, y)$, we have

$$
{ }^{\mathrm{sc}} T^{*} \bar{X}=\operatorname{span}\left\{\frac{\mathrm{d} x}{x^{2}}, \frac{\mathrm{d} y}{x}\right\}=\operatorname{span}\left\{\mathrm{d}\left(\frac{1}{x}\right), \frac{\mathrm{d} y}{x}\right\} .
$$

Thus for any $\alpha \in{ }^{\mathrm{sc}} T^{*} \bar{X}$ it can be written as

$$
\alpha=\tau \mathrm{d}\left(\frac{1}{x}\right)+\mu \cdot \frac{\mathrm{d} y}{x},
$$

and this gives linear coordinates $(\tau, \mu) \in \mathbb{R} \times \mathbb{R}^{n-1}$ on each fiber of ${ }^{\mathrm{sc}} T^{*} \bar{X}$. Thus this also gives linear coordinates $(x, y ; \tau, \mu)$ on ${ }^{\mathrm{sc}} T^{*} \bar{X}$ near the boundary $\partial \bar{X}$. On the other hand, if $(\xi, \eta)$ is the dual cotangent variables to $(x, y)$, then

$$
\alpha=\xi \mathrm{d} x+\eta \cdot \mathrm{d} y
$$

which implies $\tau=x^{2} \xi, \mu=x \eta$. We say $(\tau, \mu)$ as rescaled cotangent variables. Hence this space of operators can be microlocalized by introducing scattering pseudodifferential operators which are formally objects given by $b\left(x, y, x^{2} \partial_{x}, x \partial_{y}\right)$ with $b(x, y, \tau, \mu)$ a Kohn-Nirenberg symbol on the bundle ${ }^{\mathrm{sc}} T^{*} \bar{X}$.

In the above coordinates, the Laplacian can be written as

$$
\Delta_{g}=\sum_{j, k=1}^{n} \frac{1}{\sqrt{|g|}} \partial_{j} g^{j, k} \sqrt{|g|} \partial_{k},
$$

where $|g|$ is the determinant of the metric $g_{j k}$. To compare with the Euclidean space near the boundary, we write the metric near the boundary in the form $d r^{2}+r^{2} h\left(x, y, d y, r^{-2} d r\right)$ with respect to the local coordinates $r=1 / x$ and $y$. Then the metric components satisfy

$$
\begin{aligned}
& g_{00}=1+O\left(r^{-2}\right), g_{0, j}=O(1), g_{k j}=r^{2}\left(\tilde{h}_{k, j}+O\left(r^{-1}\right)\right) \\
& g^{00}=1+O\left(r^{-2}\right), g^{0, j}=O\left(r^{-2}\right), g^{k j}=r^{-2}\left(\tilde{h}^{k, j}+O\left(r^{-1}\right)\right),
\end{aligned}
$$


where $\tilde{h}$ is the induced metric on the boundary. Note that the cross term, with $j=0$ and $k \neq 0$ or $j \neq 0$ and $k=0$, vanishes as $x^{3}$ when expressed in terms of $x \partial_{x}$ and $\partial_{y}$ (the components in $\mathcal{V}_{b}$ ). Hence near the boundary we write

$$
\Delta_{g}=\left(x^{2} \partial_{x}\right)^{2}+(n-1) x^{3} \partial_{x}+x^{2} \Delta_{h}+x^{3} \operatorname{Diff}_{b}^{2}(\bar{X}),
$$

where $\operatorname{Diff}_{b}^{2}$ is the second-order differential b-operator. In this sense, the Laplacian on this setting is a sc-differential operator. To see more results about its resolvent and calculus, we refer to [25,33].

\subsection{The Microlocalized Spectral Measure}

In the free Euclidean space, the Klein-Gordon propagator can be written in an explicit formula by using the Fourier transform, but in our setting it turns out to be quite complicated. From the results of $[19,23]$, we have known that the Schwartz kernel of the spectral measure can be described as a Legendrian distribution on the compactification of the space $\bar{X} \times \bar{X}$ uniformly with respect to the spectral parameter $\lambda$. As pointed out in introduction, we really need to choose an operator partition of unity to microlocalize the spectral measure such that the spectral measure can be expressed in a formula capturing not only the size but also the oscillatory behavior. This was constructed and proved in [26]. For convenience, we recall it here.

Proposition 2.1 Let $(X, g)$ and $\mathrm{H}=-\Delta_{g}$ be in Theorem 1.1. Then there exists a $\lambda$-dependent scattering pseudodifferential operator partition of unity on $L^{2}(M)$

$$
\mathrm{Id}=\sum_{j=1}^{N} Q_{j}(\lambda)
$$

with $N$ independent of $\lambda$, such that for each $1 \leqslant j \leqslant N$ we can write

$$
\left(Q_{j}(\lambda) d E_{\sqrt{\mathrm{H}}}(\lambda) Q_{j}^{*}(\lambda)\right)\left(z, z^{\prime}\right)=\lambda^{n-1}\left(\sum_{ \pm} e^{ \pm i \lambda d\left(z, z^{\prime}\right)} a_{ \pm}\left(\lambda, z, z^{\prime}\right)+b\left(\lambda, z, z^{\prime}\right)\right),
$$

with estimates

$$
\begin{aligned}
& \left|\partial_{\lambda}^{\alpha} a_{ \pm}\left(\lambda, z, z^{\prime}\right)\right| \leqslant C_{\alpha} \lambda^{-\alpha}\left(1+\lambda d\left(z, z^{\prime}\right)\right)^{-\frac{n-1}{2}}, \\
& \left|\partial_{\lambda}^{\alpha} b\left(\lambda, z, z^{\prime}\right)\right| \leqslant C_{\alpha, M} \lambda^{-\alpha}\left(1+\lambda d\left(z, z^{\prime}\right)\right)^{-K} \text { for any } K .
\end{aligned}
$$

Here $d(\cdot, \cdot)$ is the Riemannian distance on $X$.

From this proposition, we can exploit the oscillations both in the multiplier $e^{i(t-s) \sqrt{1+\lambda^{2}}}$ and in $e^{ \pm i \lambda d\left(z, z^{\prime}\right)}$ to obtain the required dispersive estimate for the $T T^{*}$ version of the microlocalized propagator. 


\subsection{The Littlewood-Paley Squarefunction Estimate}

In [47], we showed the Gaussian upper bounds on the heat kernel by using the localin-time heat kernel bounds in Cheng-Li-Yau [12], and Guillarmou-Hassell-Sikora's [20] restriction estimate for low frequency. Hence we finally proved the LittlewoodPaley squarefunction estimate on this setting by using a spectral multiplier estimate in Alexopoulos [1] and Stein's [41] classical argument involving Rademacher functions. Now we recall the result here for convenience.

Let $\phi \in C_{0}^{\infty}(\mathbb{R} \backslash\{0\})$ take values in $[0,1]$ and be supported in $[1 / 2,2]$ such that

$$
1=\sum_{j \in \mathbb{Z}} \phi\left(2^{-j} \lambda\right), \quad \lambda>0 .
$$

Define $\phi_{0}(\lambda)=\sum_{j \leqslant 0} \phi\left(2^{-j} \lambda\right)$. The result about the Littlewood-Paley squarefunction estimate reads as follows:

Proposition 2.2 Let $(X, g)$ be a scattering manifold, trapping or not, and $\mathrm{H}=-\Delta_{g}$ is the Laplace-Beltrami operator on $(X, g)$. Then for $1<p<\infty$, there exist constants $c_{p}$ and $C_{p}$ depending on $p$ such that

$$
c_{p}\|f\|_{L^{p}(X)} \leqslant\left\|\left(\sum_{j \in \mathbb{Z}}\left|\phi\left(2^{-j} \sqrt{\mathrm{H}}\right) f\right|^{2}\right)^{\frac{1}{2}}\right\|_{L^{p}(X)} \leqslant C_{p}\|f\|_{L^{p}(X)} .
$$

One important application of the traditional Littlewood-Paley theory is the proof of Leibniz (=product) and chain rules for differential operators of noninteger order. For example, if $1<p, p_{j}<\infty$ with $j=1, \ldots 4$ and $s>0$, then

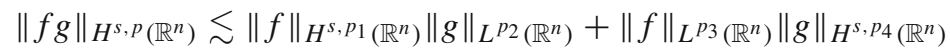

whenever $\frac{1}{p}=\frac{1}{p_{1}}+\frac{1}{p_{2}}=\frac{1}{p_{3}}+\frac{1}{p_{4}}$. For a textbook presentation of these theorems and original references, see [44]. The Leibniz chain rules is a basic tool in the proof of well-posedness. Since we have heat kernel estimate with Gaussian upper bounds and the Littlewood-Paley squarefunction estimate, the Leibniz chain rules can be obtained by similar argument in Euclidean space and it also was proved in [14, Theorem 27]. We record here

Proposition 2.3 Let $H^{s, p}(X)=\left(1-\Delta_{g}\right)^{-\frac{s}{2}} L^{p}(X)$ be the inhomogeneous Sobolev space over $X$. Then we have for $0 \leqslant s \leqslant 1$

$$
\|f g\|_{H^{s, p}(X)} \lesssim\|f\|_{H^{s, p_{1}(X)}}\|g\|_{L^{p_{2}(X)}}+\|f\|_{L^{p_{3}(X)}}\|g\|_{H^{s, p_{4}(X)}},
$$

where $1<p, p_{j}<\infty$ with $j=1, \ldots 4$ such that $\frac{1}{p}=\frac{1}{p_{1}}+\frac{1}{p_{2}}=\frac{1}{p_{3}}+\frac{1}{p_{4}}$. 


\section{$3 L^{2}$-Estimates and Dispersive Estimates}

In this section, we prove the $L^{2}$-estimates for $U_{j, k}(t)$ and dispersive estimates for $U_{j, k}(t) U_{j, k}^{*}(s)$ where $U_{j, k}(t)$ is a microlocalized Klein-Gordon propagator. The $L^{2}$ estimate is showed by the spectral theory on Hilbert space. The conjugate points are separated in the microlocalized propagators, and hence we can prove the $T T^{*}$ version dispersive estimates. Since the abstract Klein-Gordon propagator $U(t)=e^{i t} \sqrt{1-\Delta_{g}}$ behaves like the Schrödinger at low frequency and wave at high frequency, we need to establish dispersive estimate by using different arguments at different frequencies.

\subsection{Microlocalized Propagator}

We start by dividing the Klein-Gordon propagator into a low-energy piece and a high-energy piece. Using the dyadic partition of unity $1=\sum_{k \in \mathbb{Z}} \phi\left(2^{-k} \lambda\right)$ we further define

$$
U_{k}(t)=\int_{0}^{\infty} e^{i t \sqrt{1+\lambda^{2}}} \phi\left(2^{-k} \lambda\right) d E_{\sqrt{\mathrm{H}}}(\lambda), \quad k \in \mathbb{Z}
$$

Further using scattering pseudodifferential operator partition of identity operator in Proposition 2.1, we define

$$
U_{j, k}(t)=\int_{0}^{\infty} e^{i t \sqrt{1+\lambda^{2}}} \phi\left(2^{-k} \lambda\right) Q_{j}(\lambda) \mathrm{d} E_{\sqrt{\mathrm{H}}}(\lambda), \quad 1 \leqslant j \leqslant N, k \in \mathbb{Z} .
$$

We divide the microlocalized Klein-Gordon propagator into low frequency and high frequency

$$
\begin{array}{r}
U_{j}^{\text {low }}(t)=\int_{0}^{\infty} e^{i t \sqrt{1+\lambda^{2}}} \phi_{0}(\lambda) Q_{j}(\lambda) \mathrm{d} E_{\sqrt{\mathrm{H}}}(\lambda), \quad 1 \leqslant j \leqslant N ; \\
U_{j}^{\text {high }}(t)=\sum_{k=0}^{\infty} \int_{0}^{\infty} e^{i t \sqrt{1+\lambda^{2}}} \phi\left(2^{-k} \lambda\right) Q_{j}(\lambda) \mathrm{d} E_{\sqrt{\mathrm{H}}}(\lambda), \quad 1 \leqslant j \leqslant N .
\end{array}
$$

\section{$3.2 L^{2}$-Estimate for $U_{j, k}(t)$.}

In this subsection we show this definition is well defined and prove $U_{j, k}(t)$ is a bounded operator on $L^{2}(X)$. Essentially this has been proved in [47, Proposition 3.2]. For convenience, we sketch it here. Indeed it suffices to show the above integrals are well defined over any compact interval in $(0, \infty)$. Let $A(\lambda)=e^{i t \sqrt{1+\lambda^{2}}} \phi\left(2^{-k}\right) Q_{j}(\lambda)$. Then $A(\lambda)$ is a family of bounded operators on $L^{2}(X)$, compactly supported in $\left[2^{k-1}, 2^{k+1}\right]$ and $\mathcal{C}^{1}$ in $\lambda \in(0, \infty)$. Integrating by parts, the integral of

$$
\int_{2^{k-1}}^{2^{k+1}} A(\lambda) \mathrm{d} E_{\sqrt{\mathrm{H}}}(\lambda)
$$


is given by

$$
E_{\sqrt{\mathrm{H}}}\left(2^{k+1}\right) A\left(2^{k+1}\right)-E_{\sqrt{\mathrm{H}}}\left(2^{k-1}\right) A\left(2^{k-1}\right)-\int_{2^{k-1}}^{2^{k+1}} \frac{\mathrm{d}}{\mathrm{d} \lambda} A(\lambda) E_{\sqrt{\mathrm{H}}}(\lambda) \mathrm{d} \lambda .
$$

Hence the operators $U_{j, k}(t)$ are well defined by using the following lemma which is the consequence of [26, Lemmas 2.3, 3.1].

Lemma 3.1 Each $Q_{j}(\lambda)$ and each operator $\lambda \partial_{\lambda} Q_{j}(\lambda)$ is bounded on $L^{2}(X)$ uniformly in $\lambda$.

Since $\left\|U_{j, k}\right\|_{L^{2} \rightarrow L^{2}} \leqslant C$ is equivalent to $\left\|U_{j, k} U_{j, k}^{*}\right\|_{L^{2} \rightarrow L^{2}} \leqslant C$, we compute by [26, Lemma 5.3],

$$
\begin{aligned}
U_{j, k}(t) U_{j, k}(t)^{*}= & \int \phi\left(\frac{\lambda}{2^{k}}\right) \phi\left(\frac{\lambda}{2^{k}}\right) Q_{j}(\lambda) d E_{\sqrt{\mathrm{H}}}(\lambda) Q_{j}(\lambda)^{*} \\
= & -\int \frac{d}{d \lambda}\left(\phi\left(\frac{\lambda}{2^{k}}\right) \phi\left(\frac{\lambda}{2^{k}}\right) Q_{j}(\lambda)\right) E_{\sqrt{\mathrm{H}}}(\lambda) Q_{j}(\lambda)^{*} \\
& -\int \phi\left(\frac{\lambda}{2^{k}}\right) \phi\left(\frac{\lambda}{2^{k}}\right) Q_{j}(\lambda) E_{\sqrt{\mathrm{H}}}(\lambda) \frac{\mathrm{d}}{\mathrm{d} \lambda} Q_{j}(\lambda)^{*}
\end{aligned}
$$

We observe that this is independent of $t$ and we also note that the integrand is a bounded operator on $L^{2}$, with an operator bound of the form $C / \lambda$ where $C$ is uniform, as we see from Lemma 3.1 and the support property of $\phi$. The integral is therefore uniformly bounded, as we are integrating over a dyadic interval in $\lambda$. Hence we have shown that

Proposition 3.2 ( $L^{2}$-estimates) Let $U_{j, k}(t)$ be defined in (3.2). Then there exists a constant $C$ independent of $t, z, z^{\prime}$ such that $\left\|U_{j, k}(t)\right\|_{L^{2} \rightarrow L^{2}} \leqslant C$ for all $j \geqslant 1, k \in \mathbb{Z}$.

Since there is no difference between $e^{i t \lambda^{2}}$ and $e^{i t \sqrt{1+\lambda^{2}}}$ in the proof [26, Proposition 5.1] (using a almost orthogonal property in the summation of $k$ ), we have

Proposition 3.3 ( $L^{2}$-estimates) Let $U_{j}^{\text {low }}(t)$ be defined in (3.3). Then there exists a constant $C$ independent of $t, z, z^{\prime}$ such that $\left\|U_{j}^{\text {low }}(t)\right\|_{L^{2} \rightarrow L^{2}} \leqslant C$ for all $j \geqslant 1$.

\subsection{Dispersive Estimates}

In this subsection, we use stationary phase argument and Proposition 2.1 to establish the microlocalized dispersive estimates. Before doing this, we prove a fundamental result on decay estimate.

Proposition 3.4 (Microlocalized dispersive estimates for low frequency) Let $Q_{j}(\lambda)$ be in Proposition 2.1. Then for all integers $j \geqslant 1$, the kernel estimate

$$
\left|\int_{0}^{\infty} e^{i t \sqrt{1+\lambda^{2}}} \phi_{0}(\lambda)\left(Q_{j}(\lambda) \mathrm{d} E_{\sqrt{\mathrm{H}}}(\lambda) Q_{j}^{*}(\lambda)\right)\left(z, z^{\prime}\right) \mathrm{d} \lambda\right| \leqslant C(1+|t|)^{-\frac{n}{2}}
$$


holds for a constant $C$ independent of points $z, z^{\prime} \in X$.

Proof The key things in the proof are to use the property of spectral measure in Proposition 2.1 and stationary phase argument. When $|t| \lesssim 1$, it is easy to show it due to the compact support of $\phi_{0}$. From now on, we only need to consider the case $t \gg 1$ by symmetry. Let $r=d\left(z, z^{\prime}\right)$ and $\bar{r}=r t^{-\frac{1}{2}}$. In this case, we write the kernel using Proposition 2.1

$$
\begin{aligned}
& \int_{0}^{\infty} e^{i t \sqrt{1+\lambda^{2}}} \phi_{0}(\lambda)\left(Q_{j}(\lambda) \mathrm{d} E_{\sqrt{\mathrm{H}}}(\lambda) Q_{j}^{*}(\lambda)\right)\left(z, z^{\prime}\right) \mathrm{d} \lambda \\
& =\sum_{ \pm} \int_{0}^{\infty} e^{i t \sqrt{1+\lambda^{2}}} e^{ \pm i r \lambda} \lambda^{n-1} \phi_{0}(\lambda) a_{ \pm}\left(\lambda, z, z^{\prime}\right) \mathrm{d} \lambda \\
& \quad+\int_{0}^{\infty} e^{i t \sqrt{1+\lambda^{2}}} \lambda^{n-1} \phi_{0}(\lambda) b\left(\lambda, z, z^{\prime}\right) \mathrm{d} \lambda \\
& =t^{-\frac{n}{2}} \sum_{ \pm} \int_{0}^{\infty} e^{i \sqrt{t^{2}+t \lambda^{2}}} e^{ \pm i \bar{r} \lambda} \lambda^{n-1} \phi_{0}\left(t^{-1 / 2} \lambda\right) a_{ \pm}\left(t^{-1 / 2} \lambda, z, z^{\prime}\right) \mathrm{d} \lambda \\
& \quad+\int_{0}^{\infty} e^{i t \sqrt{1+\lambda^{2}}} \lambda^{n-1} \phi_{0}(\lambda) b\left(\lambda, z, z^{\prime}\right) \mathrm{d} \lambda,
\end{aligned}
$$

where $a_{ \pm}$satisfies estimates

$$
\left|\partial_{\lambda}^{\alpha} a_{ \pm}\left(\lambda, z, z^{\prime}\right)\right| \leqslant C_{\alpha} \lambda^{-\alpha}\left(1+\lambda \mathrm{d}\left(z, z^{\prime}\right)\right)^{-\frac{n-1}{2}},
$$

and therefore

$$
\left|\partial_{\lambda}^{\alpha}\left(a_{ \pm}\left(t^{-1 / 2} \lambda, z, z^{\prime}\right)\right)\right| \leqslant C_{\alpha} \lambda^{-\alpha}(1+\lambda \bar{r})^{-\frac{n-1}{2}}
$$

First, we show the contribution of the above term with $b\left(\lambda, z, z^{\prime}\right)$. We can use the estimate (2.7) to obtain

$$
\left|\left(\frac{\mathrm{d}}{\mathrm{d} \lambda}\right)^{N} b\left(\lambda, z, z^{\prime}\right)\right| \leqslant C_{N} \lambda^{n-1-N} \quad \forall N \in \mathbb{N} .
$$

Let $\delta$ be a small constant to be chosen later. Recall that we chose $\phi \in C_{c}^{\infty}\left(\left[\frac{1}{2}, 2\right]\right)$ such that $\sum_{m \in \mathbb{Z}} \phi\left(2^{-m} \lambda\right)=1$; we denote $\phi_{0}(\lambda)=\sum_{m \leqslant-1} \phi\left(2^{-m} \lambda\right)$. Then

$$
\left|\int_{0}^{\infty} e^{i t \sqrt{1+\lambda^{2}}} b\left(\lambda, z, z^{\prime}\right) \phi_{0}(\lambda) \phi_{0}\left(\frac{\lambda}{\delta}\right) \mathrm{d} \lambda\right| \leqslant C \int_{0}^{\delta} \lambda^{n-1} \mathrm{~d} \lambda \leqslant C \delta^{n} .
$$

We use integration by parts $N$ times to obtain, using (3.9) 


$$
\begin{aligned}
& \left|\int_{0}^{\infty} e^{i t \sqrt{1+\lambda^{2}}} \phi_{0}(\lambda) \sum_{m \geqslant 0} \phi\left(\frac{\lambda}{2^{m} \delta}\right) b\left(\lambda, z, z^{\prime}\right) \mathrm{d} \lambda\right| \\
& \quad \leqslant \sum_{m \geqslant 0}\left|\int_{0}^{\infty}\left(\frac{\sqrt{1+\lambda^{2}}}{i \lambda t} \frac{\partial}{\partial \lambda}\right)^{N}\left(e^{i t \sqrt{1+\lambda^{2}}}\right) \phi_{0}(\lambda) \phi\left(\frac{\lambda}{2^{m} \delta}\right) b\left(\lambda, z, z^{\prime}\right) \mathrm{d} \lambda\right| \\
& \quad \leqslant C_{N}|t|^{-N} \sum_{m \geqslant 0} \int_{2^{m-1} \delta}^{2^{m+1} \delta} \lambda^{n-1-2 N} \mathrm{~d} \lambda \leqslant C_{N}|t|^{-N} \delta^{n-2 N} .
\end{aligned}
$$

Choosing $\delta=|t|^{-\frac{1}{2}}$, we have thus proved

$$
\left|\int_{0}^{\infty} e^{i t \sqrt{1+\lambda^{2}}} \phi_{0}(\lambda) b\left(\lambda, z, z^{\prime}\right) \mathrm{d} \lambda\right| \leqslant C_{N}|t|^{-\frac{n}{2}} .
$$

Now we consider first term in RHS of (3.7). We divide it into two pieces using the partition of unity above. It suffices to prove that there exists a constant $C$ independent of $\bar{r}$ such that

$$
\begin{aligned}
I^{ \pm} & :=\left|\int_{0}^{\infty} e^{i \sqrt{t^{2}+t \lambda^{2}}} e^{ \pm i \bar{r} \lambda} \lambda^{n-1} \phi_{0}\left(t^{-1 / 2} \lambda\right) a_{ \pm}\left(t^{-1 / 2} \lambda, z, z^{\prime}\right) \phi_{0}(\lambda) \mathrm{d} \lambda\right| \leqslant C, \\
I^{ \pm} & :=\left|\sum_{m \geqslant 0} \int_{0}^{\infty} e^{i \sqrt{t^{2}+t \lambda^{2}}} e^{ \pm i \bar{r} \lambda} \lambda^{n-1} \phi_{0}\left(t^{-1 / 2} \lambda\right) a_{ \pm}\left(t^{-1 / 2} \lambda, z, z^{\prime}\right) \phi\left(\frac{\lambda}{2^{m}}\right) \mathrm{d} \lambda\right| \leqslant C .
\end{aligned}
$$

The estimate for $I^{ \pm}$is obvious, since $\lambda \leqslant 1$. For $I I^{+}$, we use integration by parts. Notice that

$$
L^{+}\left(e^{i \sqrt{t^{2}+t \lambda^{2}}+i \bar{r} \lambda}\right)=e^{i \sqrt{t^{2}+t \lambda^{2}}+i \bar{r} \lambda}, \quad L^{+}=\frac{-i}{\frac{t \lambda}{\sqrt{t^{2}+t \lambda^{2}}}+\bar{r}} \frac{\partial}{\partial \lambda} .
$$

Note that if $0<\lambda<\sqrt{t}$, we have for $k \geqslant 0$ by induction

$$
\partial_{\lambda}^{k}\left[\left(\frac{t \lambda}{\sqrt{t^{2}+t \lambda^{2}}}+\bar{r}\right)^{-1}\right] \leqslant C_{k} \lambda^{-1-k}
$$

Writing

$$
e^{i \sqrt{t^{2}+t \lambda^{2}}+i \bar{r} \lambda}=\left(L^{+}\right)^{N}\left(e^{i \sqrt{t^{2}+t \lambda^{2}}+i \bar{r} \lambda}\right)
$$

and integrating by parts, we gain a factor of $\lambda^{-2 N}$ thanks to (3.16) and (3.11). Thus $I^{+}$can be estimated by

$$
\sum_{m \geqslant 0} \int_{\lambda \sim 2^{m}} \lambda^{n-1-2 N} \mathrm{~d} \lambda \leqslant C
$$


To treat $I I^{-}$, we introduce a further decomposition, based on the size of $\bar{r} \lambda$. We write $I I^{-}=I I_{1}^{-}+I I_{2}^{-}$, where (dropping the-superscripts and subscripts from here on)

$$
\begin{aligned}
I_{1}= & \mid \sum_{m \geqslant 0} \int_{0}^{\infty} e^{i \sqrt{t^{2}+t \lambda^{2}}} e^{-i \bar{r} \lambda} \lambda^{n-1} \phi_{0}\left(t^{-1 / 2} \lambda\right) a\left(t^{-1 / 2} \lambda, z, z^{\prime}\right) \\
& \phi\left(\frac{\lambda}{2^{m}}\right) \phi_{0}(8 \bar{r} \lambda) \mathrm{d} \lambda \mid, \\
I I_{2}= & \mid \int_{0}^{\infty} e^{i \sqrt{t^{2}+t \lambda^{2}}} e^{-i \bar{r} \lambda} \lambda^{n-1} \phi_{0}\left(t^{-1 / 2} \lambda\right) a\left(t^{-1 / 2} \lambda, z, z^{\prime}\right)\left(1-\phi_{0}(\lambda)\right) \\
& \left(1-\phi_{0}(8 \bar{r} \lambda)\right) \mathrm{d} \lambda \mid .
\end{aligned}
$$

Let $\Phi(\lambda, \bar{r})=\sqrt{t^{2}+t \lambda^{2}}-\bar{r} \lambda$. We first consider $I I_{1}$. Since the integral for $I I_{1}$ is supported where $\lambda \leqslant(8 \bar{r})^{-1}$ and $\lambda \geqslant 1 / 2$, the integrand is only nonzero when $\bar{r} \leqslant 1 / 4$. Since $\lambda<\sqrt{t}$, therefore $\left|\partial_{\lambda} \Phi\right|=\frac{t \lambda}{\sqrt{t^{2}+t \lambda^{2}}}-\bar{r} \geqslant \frac{\sqrt{2}}{2} \lambda-\bar{r} \geqslant \frac{1}{10} \lambda$. Define the operator $L=L(\lambda, \bar{r})=\left(\frac{t \lambda}{\sqrt{t^{2}+t \lambda^{2}}}-\bar{r}\right)^{-1} \partial_{\lambda}$. On the support of $\phi_{0}(\lambda / \sqrt{t})$, we have for $k \geqslant 0$

$$
\partial_{\lambda}^{k}\left[\left(\frac{t \lambda}{\sqrt{t^{2}+t \lambda^{2}}}-\bar{r}\right)^{-1}\right] \leqslant C_{k} \lambda^{-1-k} .
$$

By (3.16) and using integration by parts, we obtain for $N>n / 2$

$$
\begin{aligned}
I_{1} \leqslant & \sum_{m \geqslant 0} \mid \int_{0}^{\infty} e^{i \sqrt{t^{2}+t \lambda^{2}}} e^{-i \bar{r} \lambda} \lambda^{n-1} \phi_{0}\left(t^{-1 / 2} \lambda\right) \\
& a\left(t^{-1 / 2} \lambda, z, z^{\prime}\right) \phi\left(\frac{\lambda}{2^{m}}\right) \phi_{0}(8 \bar{r} \lambda) \mathrm{d} \lambda \mid \\
= & \sum_{m \geqslant 0} \mid \int_{0}^{\infty} L^{N}\left(e^{i\left(\frac{\lambda}{\sqrt{t^{2}+\lambda^{2}}}-\bar{r} \lambda\right)}\right)\left[\lambda^{n-1} \phi_{0}\left(t^{-1 / 2} \lambda\right)\right. \\
& \left.a\left(t^{-1 / 2} \lambda, z, z^{\prime}\right) \phi\left(\frac{\lambda}{2^{m}}\right) \phi_{0}(8 \bar{r} \lambda)\right] \mathrm{d} \lambda \mid \\
\leqslant & C_{N} \sum_{m \geqslant 0} \int_{|\lambda| \sim 2^{m}} \lambda^{n-1-2 N} \mathrm{~d} \lambda \leqslant C_{N} .
\end{aligned}
$$

Finally we consider $I I_{2}$. Here, we replace the decomposition $\sum_{m} \phi\left(2^{-m} \lambda\right)$ with a different decomposition, based on the size of $\partial_{\lambda} \Phi$.

$$
\begin{aligned}
I_{2} \leqslant & \mid \int_{0}^{\infty} e^{i \sqrt{t^{2}+t \lambda^{2}}} e^{-i \bar{r} \lambda} \lambda^{n-1} \phi_{0}\left(t^{-1 / 2} \lambda\right) a\left(t^{-1 / 2} \lambda, z, z^{\prime}\right) \\
& \times\left(1-\phi_{0}(\lambda)\right) \phi_{0}\left(\frac{t \lambda}{\sqrt{t^{2}+t \lambda^{2}}}-\bar{r}\right)\left(1-\phi_{0}(8 \bar{r} \lambda)\right) \mathrm{d} \lambda \mid
\end{aligned}
$$




$$
\begin{aligned}
& \quad+\sum_{m \geqslant 0} \mid \int_{0}^{\infty} e^{i \sqrt{t^{2}+t \lambda^{2}}} e^{-i \bar{r} \lambda} \lambda^{n-1} \phi_{0}\left(t^{-1 / 2} \lambda\right) a\left(t^{-1 / 2} \lambda, z, z^{\prime}\right) \\
& \quad \times\left(1-\phi_{0}(\lambda)\right) \phi\left(\frac{\frac{t \lambda}{\sqrt{t^{2}+t \lambda^{2}}}-\bar{r}}{2^{m}}\right)\left(1-\phi_{0}(8 \bar{r} \lambda)\right) \mathrm{d} \lambda \mid \\
& :=I I_{2}^{1}+I I_{2}^{2} .
\end{aligned}
$$

If $\bar{r} \leqslant 10$, note $\lambda<\sqrt{t}$ again, then for the integrand of $I I_{2}^{1}$ to be nonzero we must have $\lambda \leqslant 100$, due to the second $\phi_{0}$ factor in $I I_{2}^{1}$. Then it is easy to see that $I I_{2}^{1}$ is uniformly bounded. If $\bar{r} \geqslant 10$, by $\left|\frac{t \lambda}{\sqrt{t^{2}+t \lambda^{2}}}-\bar{r}\right| \leqslant 1$ and $\lambda<\sqrt{t}$, we have $\bar{r} \sim \lambda$. Hence, using (3.16) with $\alpha=0$,

$$
\begin{aligned}
I I_{2}^{1} & \leq \int_{\left\{\lambda<\sqrt{t}:\left|\frac{t \lambda}{\sqrt{t^{2}+t \lambda^{2}}}-\bar{r}\right| \leqslant 1\right\}} \lambda^{n-1}(1+\bar{r} \lambda)^{-\frac{n-1}{2}} \mathrm{~d} \lambda \\
& \leqslant C t^{1 / 2} \int_{\left\{\lambda<1:\left|\frac{\lambda}{\sqrt{1+\lambda^{2}}}-\frac{\bar{r}}{\sqrt{t}}\right| \leqslant 1 / \sqrt{t}\right\}} \mathrm{d} \lambda \\
& \leqslant C t^{1 / 2} \int_{\left\{\lambda<1:\left|\bar{\lambda}-\frac{\bar{r}}{\sqrt{t}}\right| \leqslant 1 / \sqrt{t}\right\}}\left(1+\lambda^{2}\right)^{3 / 2} \mathrm{~d} \bar{\lambda} \leqslant C .
\end{aligned}
$$

Now we consider the second term. We write

$$
\begin{aligned}
I I_{2}^{2} \leqslant \sum_{m \geqslant 0} \mid \int_{0}^{\sqrt{t}} e^{i \sqrt{t^{2}+t \lambda^{2}}} e^{-i \bar{r} \lambda} \phi_{0}\left(t^{-1 / 2} \lambda\right) \lambda^{n-1} a\left(t^{-1 / 2} \lambda, z, z^{\prime}\right) \\
\quad \times\left(1-\phi_{0}(\lambda)\right) \phi\left(\frac{\frac{t \lambda}{\sqrt{t^{2}+t \lambda^{2}}}-\bar{r}}{2^{m}}\right)\left(1-\phi_{0}(8 \bar{r} \lambda)\right) \mathrm{d} \lambda \mid \\
=\sum_{m \geqslant 0} \mid \int L^{N}\left(e^{i\left(\sqrt{t^{2}+t \lambda^{2}}-\bar{r} \lambda\right)}\right)\left[\phi_{0}\left(t^{-1 / 2} \lambda\right) \lambda^{n-1} a\left(t^{-1 / 2} \lambda, z, z^{\prime}\right)\right. \\
\left.\quad \times\left(1-\phi_{0}(\lambda)\right) \phi\left(\frac{\frac{t \lambda}{\sqrt{t^{2}+t \lambda^{2}}}-\bar{r}}{2^{m}}\right)\left(1-\phi_{0}(8 \bar{r} \lambda)\right)\right] \mathrm{d} \lambda \mid .
\end{aligned}
$$

Let

$$
b(\lambda)=\lambda^{n-1} a\left(t^{-1 / 2} \lambda, z, z^{\prime}\right)\left(1-\phi_{0}(\lambda)\right) \phi\left(\frac{\frac{t \lambda}{\sqrt{t^{2}+t \lambda^{2}}}-\bar{r}}{2^{m}}\right)\left(1-\phi_{0}(8 \bar{r} \lambda)\right),
$$

then we have the rough estimate, due to the support of $b$

$$
\left|\partial_{\lambda}^{\alpha} b\right| \leqslant C_{\alpha} \lambda^{n-1}(1+\bar{r} \lambda)^{-(n-1) / 2} .
$$


Hence we obtain

$$
\left|\left(L^{*}\right)^{N}[b(\lambda)]\right| \leqslant C_{N} 2^{-m N} \lambda^{n-1}(1+\bar{r} \lambda)^{-(n-1) / 2} .
$$

Therefore we obtain by using integrating by parts and (3.16)

$$
I I_{2}^{2} \leqslant C_{N} \sum_{m \geqslant 0} 2^{-m N} \int_{\left\{\lambda<\sqrt{t},\left|\frac{t \lambda}{\sqrt{t^{2}+t \lambda^{2}}}-\bar{r}\right| \sim 2^{m}\right\}} \lambda^{n-1}(1+\bar{r} \lambda)^{-\frac{n-1}{2}} \mathrm{~d} \lambda .
$$

If $\bar{r} \leqslant 2^{m+1}$, then $\lambda \leqslant 2^{m+2}$ on the support of the integrand.

$$
I I_{2}^{2} \leqslant C_{N} \sum_{m \geqslant 0} 2^{-m N} 2^{(m+2) n} \leqslant C .
$$

If $\bar{r} \geqslant 2^{m+1}$, we have $\lambda \sim \bar{r}$, thus

$$
I I_{2}^{2} \leqslant C_{N} t^{1 / 2} \sum_{m \geqslant 0} 2^{-m N} \int_{\left\{\lambda<1:\left|\frac{\lambda}{\sqrt{1+\lambda^{2}}}-\frac{\bar{r}}{\sqrt{t}}\right| \sim \frac{2^{m}}{\sqrt{t}}\right\}} d \lambda \leqslant C_{N} \sum_{m \geqslant 0} 2^{-m N} 2^{m},
$$

which is summable for $N>1$. Therefore we have completed the proof of Proposition 3.4 .

Proposition 3.5 (Microlocalized dispersive estimates for high frequency) Let $Q_{j}(\lambda)$ be in Proposition 2.1. Then for all integers $j \geqslant 1$ and $k \geqslant 0$, the kernel estimate

$$
\begin{aligned}
\left|\int_{0}^{\infty} e^{i t \sqrt{1+\lambda^{2}}} \phi\left(2^{-k} \lambda\right)\left(Q_{j}(\lambda) d E_{\sqrt{\mathrm{H}}}(\lambda) Q_{j}^{*}(\lambda)\right)\left(z, z^{\prime}\right) d \lambda\right| \\
\leqslant C 2^{k(n+1+\theta) / 2}\left(2^{-k}+|t|\right)^{-(n-1+\theta) / 2}
\end{aligned}
$$

holds for $0 \leqslant \theta \leqslant 1$ and a constant $C$ independent of $k$ and points $z, z^{\prime} \in X$.

Proof Let $h=2^{-k} \leqslant 1$. The key to the proof is to use the estimates in Proposition 2.1. If $|t| \leqslant h$, it is easy to see (3.13) due to

$$
\left|Q_{j}(\lambda) \mathrm{d} E_{\sqrt{\mathrm{H}}}(\lambda) Q_{j}^{*}(\lambda)\right| \leqslant C \lambda^{n-1} .
$$

From now on, we only consider $|t| \geqslant h=2^{-k}$. By the scaling, this is a directly consequence of

$$
\begin{aligned}
& \left|\int_{0}^{\infty} e^{i t \sqrt{h^{2}+\lambda^{2}} / h} \phi(\lambda)\left(Q_{j} \mathrm{~d} E_{\sqrt{\mathrm{H}}} Q_{j}^{*}\right)\left(\lambda / h, z, z^{\prime}\right) \mathrm{d} \lambda\right| \\
& \quad \leqslant C h^{-(n-1)}(|t| / h)^{-\frac{n-1}{2}}(1+h|t|)^{-1 / 2} .
\end{aligned}
$$


Indeed if we have done this, we have for $0 \leqslant \theta \leqslant 1$

$$
\begin{aligned}
& \left|\int_{0}^{\infty} e^{i t \sqrt{1+\lambda^{2}}} \phi\left(2^{-k} \lambda\right)\left(Q_{j}(\lambda) \mathrm{d} E_{\sqrt{\mathrm{H}}}(\lambda) Q_{j}^{*}(\lambda)\right)\left(z, z^{\prime}\right) \mathrm{d} \lambda\right| \\
& \quad \leqslant C 2^{k(n+1) / 2}|t|^{-(n-1) / 2}\left(1+2^{-k}|t|\right)^{-1 / 2} \\
& \quad \leqslant C 2^{k(n+1+\theta) / 2}\left(2^{-k}+|t|\right)^{-(n-1+\theta) / 2}\left(2^{-k}|t|\right)^{\frac{\theta}{2}}\left(1+2^{-k}|t|\right)^{-1 / 2}
\end{aligned}
$$

which implies (3.13).

Now we prove (3.14). Let $r=d\left(z, z^{\prime}\right)$, we write

$$
\begin{aligned}
& \mid \int_{0}^{\infty} e^{i t \sqrt{h^{2}+\lambda^{2}} / h} \phi(\lambda)\left(Q_{j} d E_{\sqrt{\mathrm{H}}} Q_{j}^{*}\right)\left(\lambda / h, z, z^{\prime}\right) \mathrm{d} \lambda \\
& =\sum_{ \pm} \int_{0}^{\infty} e^{i t \sqrt{h^{2}+\lambda^{2}} / h} e^{ \pm i r \lambda / h} \phi(\lambda)(\lambda / h)^{n-1} a_{ \pm}\left(\lambda / h, z, z^{\prime}\right) \mathrm{d} \lambda \\
& \quad+\int_{0}^{\infty} e^{i t \sqrt{h^{2}+\lambda^{2}} / h} \phi(\lambda)(\lambda / h)^{n-1} b\left(\lambda / h, z, z^{\prime}\right) \mathrm{d} \lambda,
\end{aligned}
$$

where $a_{ \pm}$satisfies estimates

$$
\left|\partial_{\lambda}^{\alpha} a_{ \pm}\left(\lambda, z, z^{\prime}\right)\right| \leqslant C_{\alpha} \lambda^{-\alpha}\left(1+\lambda d\left(z, z^{\prime}\right)\right)^{-\frac{n-1}{2}},
$$

and therefore

$$
\left|\partial_{\lambda}^{\alpha}\left(a_{ \pm}\left(h^{-1} \lambda, z, z^{\prime}\right)\right)\right| \leqslant C_{\alpha} \lambda^{-\alpha}\left(1+h^{-1} \lambda r\right)^{-\frac{n-1}{2}} .
$$

Consider the terms with the ' $b$ ' term, then we can use the estimate (2.7) to obtain

$$
\left|\left(\frac{d}{d \lambda}\right)^{N}\left(\phi(\lambda)(\lambda / h)^{n-1} b\left(\lambda / h, z, z^{\prime}\right)\right)\right| \leqslant C_{N}(\lambda / h)^{n-1} \lambda^{-N}, \quad \forall N \in \mathbb{N}
$$

Let $\delta$ be a small constant to be chosen later. Recall that we chose $\phi \in C_{c}^{\infty}\left(\left[\frac{1}{2}, 2\right]\right)$ such that $\sum_{m \in \mathbb{Z}} \phi\left(2^{-m} \lambda\right)=1$; we denote $\phi_{0}(\lambda)=\sum_{m \leqslant-1} \phi\left(2^{-m} \lambda\right)$. Then

$$
\begin{array}{rl}
\mid \int_{0}^{\infty} e^{i t \sqrt{h^{2}+\lambda^{2}} / h} \phi(\lambda)(\lambda / h)^{n-1} & b\left(\lambda / h, z, z^{\prime}\right) \phi_{0}\left(\frac{\lambda}{\delta}\right) \mathrm{d} \lambda \mid \\
& \leqslant C \int_{0}^{\delta}(\lambda / h)^{n-1} \mathrm{~d} \lambda \leqslant C h(\delta / h)^{n} .
\end{array}
$$


We use integration by parts $N$ times to obtain, using (3.9),

$$
\begin{aligned}
& \left|\int_{0}^{\infty} e^{i t \sqrt{h^{2}+\lambda^{2}} / h} \sum_{m \geqslant 0} \phi\left(\frac{\lambda}{2^{m} \delta}\right) \phi(\lambda)(\lambda / h)^{n-1} b\left(\lambda / h, z, z^{\prime}\right) \mathrm{d} \lambda\right| \\
& \leqslant \sum_{m \geqslant 0} \mid \int_{0}^{\infty}\left(\frac{h \sqrt{h^{2}+\lambda^{2}}}{\lambda t} \frac{\partial}{\partial \lambda}\right)^{N}\left(e^{i t \sqrt{h^{2}+\lambda^{2}} / h}\right) \\
& \quad \times \phi\left(\frac{\lambda}{2^{m} \delta}\right) \phi(\lambda)(\lambda / h)^{n-1} b\left(\lambda / h, z, z^{\prime}\right) \mathrm{d} \lambda \mid \\
& \leqslant C_{N}(|t| / h)^{-N} h^{-(n-1)} \sum_{m \geqslant 0} \int_{2^{m-1} \delta}^{2^{m+1} \delta} \lambda^{n-1-2 N} \mathrm{~d} \lambda \\
& \leqslant C_{N}(|t| / h)^{-N} h^{-(n-1)} \delta^{n-2 N} .
\end{aligned}
$$

Choosing $\delta=(|t| / h)^{-\frac{1}{2}}$ and noting $|t| \geqslant h$, we have thus proved

$$
\begin{aligned}
& \left|\int_{0}^{\infty} e^{i t \sqrt{h^{2}+\lambda^{2}} / h} \phi(\lambda)(\lambda / h)^{n-1} b\left(\lambda / h, z, z^{\prime}\right) \mathrm{d} \lambda\right| \\
& \quad \leqslant C h(h|t|)^{-\frac{n}{2}} \leqslant C(h|t|)^{-\frac{n-1}{2}}\left(h^{-1}|t|\right)^{-1 / 2} \\
& \quad \leqslant C(|t| h)^{-\frac{n-1}{2}}(1+h|t|)^{-1 / 2} .
\end{aligned}
$$

Next we consider the terms with $a_{ \pm}$. Without loss of generality, we consider $t \gg h$. Let $\Phi_{ \pm}(\lambda, h, r, t)=\sqrt{h^{2}+\lambda^{2}} \pm \frac{\lambda r}{t}$, it suffices to show there exists a constant $C$ independent of $r, t$, and $h$ such that

$$
\left|I_{h}^{ \pm}(t, r)\right| \leqslant C(|t| / h)^{-\frac{n-1}{2}}(1+h|t|)^{-1 / 2},
$$

where

$$
I_{h}^{ \pm}(t, r):=\int_{0}^{\infty} e^{i \frac{t}{h} \Phi_{ \pm}(\lambda, h, r, t)} \phi(\lambda) \lambda^{n-1} a_{ \pm}\left(\lambda / h, z, z^{\prime}\right) \mathrm{d} \lambda .
$$

If $r<t / 4$ or $r>2 t$, a simpler computation gives

$$
\left|\partial_{\lambda} \Phi_{ \pm}(\lambda, h, r, t)\right|=\left|\frac{\lambda}{\sqrt{h^{2}+\lambda^{2}}} \pm \frac{r}{t}\right| \geqslant 1 / 4 .
$$

It is not difficult to use the Leibniz rule to prove

Lemma 3.6 Let $L=\left(\frac{i t}{h} \partial_{\lambda} \Phi\right)^{-1} \partial_{\lambda}$ and let $L^{*}$ be its adjoint operator. Suppose that $b(\lambda)$ satisfies

$$
\left|\partial_{\lambda}^{\alpha} b(\lambda)\right| \leqslant \lambda^{n-1-|\alpha|}
$$


Then we have for any $N \geqslant 0$

$$
\left|\left(L^{*}\right)^{N}[b(\lambda)]\right| \leqslant C \lambda^{n-1-N} \sum_{j=0}^{N} \frac{(t / h)^{j}}{\left|\frac{i t}{h} \partial_{\lambda} \Phi\right|^{N+j}} .
$$

By integrating by parts and using this lemma, we obtain for $r<\frac{t}{4}$ or $r>2 t$

$$
\left|I_{h}^{ \pm}(t, r)\right| \leqslant C(|t| / h)^{-N}, \quad \forall N \geqslant 0
$$

which implies (3.19) since $t \geqslant h$. Therefore we only need consider the case $t \sim r$. A rough estimate gives

$$
\left|I_{h}^{ \pm}(t, r)\right| \leqslant \int_{0}^{\infty} \phi(\lambda) \lambda^{n-1}(1+\lambda r / h)^{-(n-1) / 2} \mathrm{~d} \lambda \leqslant C(|t| / h)^{-\frac{n-1}{2}} .
$$

Note that

$$
\left|\partial_{\lambda} \Phi_{+}(\lambda, h, r, t)\right|=\left|\frac{\lambda}{\sqrt{h^{2}+\lambda^{2}}}+\frac{r}{t}\right| \geqslant 1 / 2,
$$

and by using the same stationary phase argument again, we obtain

$$
\left|I_{h}^{+}(t, r)\right| \leqslant C(|t| / h)^{-N}, \quad \forall N \geqslant 0 .
$$

To estimate $I_{h}^{-}(t, r)$, we need the following Van der Corput lemma, see [41]

Lemma 3.7 (Van der Corput) Let $\phi$ be real-valued and smooth in $(a, b)$, and that $\left|\phi^{(k)}(x)\right| \geqslant 1$ for all $x \in(a, b)$. Then

$$
\left|\int_{a}^{b} e^{i \lambda \phi(x)} \psi(x) \mathrm{d} x\right| \leqslant c_{k} \lambda^{-1 / k}\left(|\psi(b)|+\int_{a}^{b}\left|\psi^{\prime}(x)\right| \mathrm{d} x\right)
$$

holds when $(i) k \geqslant 2$ or $(i i) k=1$ and $\phi^{\prime}(x)$ is monotonic. Here $c_{k}$ is a constant depending only on $k$.

It is easy to check for $h \leqslant 1$ and $\lambda \sim 1$

$$
\left|\partial_{\lambda}^{2} \Phi_{-}(\lambda, h, r, t)\right|=\left|\frac{h^{2}}{\sqrt{h^{2}+\lambda^{2}}}\right| \geqslant \frac{h^{2}}{100} .
$$

By using the Van der Corput lemma with $\lambda=t h$, we show

$$
\begin{aligned}
\left|I_{h}^{-}(t, r)\right| & \leqslant C(|t| / h)^{-1 / 2} \int_{0}^{\infty}\left|\frac{\mathrm{d}}{\mathrm{d} \lambda}\left(\phi(\lambda) \lambda^{n-1} a_{-}\left(\lambda / h, z, z^{\prime}\right)\right)\right| \mathrm{d} \lambda \\
& \leqslant C(|t| / h)^{-1 / 2} \int_{0}^{2} \lambda^{n-2}(1+\lambda r / h)^{-\frac{n-1}{2}} d \lambda \\
& \leqslant C(t h)^{-1 / 2}(t / h)^{-\frac{n-1}{2}} .
\end{aligned}
$$


This together with (3.23), we prove (3.19).

As two consequences of Propositions 3.4 and 3.5, respectively, we immediately have

Proposition 3.8 Let $U_{j}^{\text {low }}(t)$ be defined in (3.3). Then there exists a constant $C$ independent of $t, z, z^{\prime}$ for all $j \geqslant 1$, such that

$$
\left\|U_{j}^{\text {low }}(t)\left(U_{j}^{\text {low }}\right)^{*}(s)\right\|_{L^{1} \rightarrow L^{\infty}} \leqslant C(1+|t-s|)^{-n / 2} .
$$

Proposition 3.9 Let $U_{j, k}(t)$ be defined in (3.2). Then there exists a constant $C$ independent of $t, z, z^{\prime}$ for all $j \geqslant 1, k \in \mathbb{Z}^{+}$such that

$$
\left\|U_{j, k}(t) U_{j, k}^{*}(s)\right\|_{L^{1} \rightarrow L^{\infty}} \leqslant C 2^{k(n+1+\theta) / 2}\left(2^{-k}+|t-s|\right)^{-(n-1+\theta) / 2},
$$

where $0 \leqslant \theta \leqslant 1$.

\section{Strichartz Estimates}

In this section, we show the Strichartz estimates in Theorem 1.1. To obtain the Strichartz estimates for high frequency, we need a variant of Keel-Tao's abstract Strichartz estimate.

\subsection{Semiclassical Strichartz Estimates}

We recall a variety of the abstract Keel-Tao's Strichartz estimates theorem proved in [47], which is an analogue of the semiclassical Strichartz estimates for Schrödinger in $[30,46]$.

Proposition 4.1 Let $(X, \mathcal{M}, \mu)$ be a $\sigma$-finite measured space and $U: \mathbb{R} \rightarrow$ $B\left(L^{2}(X, \mathcal{M}, \mu)\right)$ be a weakly measurable map satisfying, for some constants $C$, $\alpha \geqslant 0, \sigma, h>0$,

$$
\begin{aligned}
\|U(t)\|_{L^{2} \rightarrow L^{2}} & \leqslant C, \quad t \in \mathbb{R}, \\
\left\|U(t) U(s)^{*} f\right\|_{L^{\infty}} & \leqslant C h^{-\alpha}(h+|t-s|)^{-\sigma}\|f\|_{L^{1}} .
\end{aligned}
$$

Then for every pair $q, r \in[1, \infty]$ such that $(q, r, \sigma) \neq(2, \infty, 1)$ and

$$
\frac{1}{q}+\frac{\sigma}{r} \leqslant \frac{\sigma}{2}, \quad q \geq 2,
$$

there exists a constant $\tilde{C}$ only depending on $C, \sigma, q$, and $r$ such that

$$
\left(\int_{\mathbb{R}}\left\|U(t) u_{0}\right\|_{L^{r}}^{q} \mathrm{~d} t\right)^{\frac{1}{q}} \leqslant \tilde{C} \Lambda(h)\left\|u_{0}\right\|_{L^{2}},
$$


where $\Lambda(h)=h^{-(\alpha+\sigma)\left(\frac{1}{2}-\frac{1}{r}\right)+\frac{1}{q}}$.

\subsection{Homogeneous Strichartz Estimates}

Now we prove the homogeneous Strichartz estimates. Using the Littlewood-Paley frequency cutoff $\phi_{m}(\sqrt{\mathrm{H}})$, we define

$$
u_{m}(t, \cdot)=\phi_{m}(\sqrt{\mathrm{H}}) u(t, \cdot) .
$$

Then the frequency-localized solutions $\left\{u_{m}\right\}_{m \in \mathbb{Z}}$ solves the family of Cauchy problems

$$
\partial_{t}^{2} u_{m}+\mathrm{H} u_{m}+u_{m}=0, \quad u_{m}(0)=f_{m}(z), \partial_{t} u_{m}(0)=g_{m}(z),
$$

where $f_{m}=\phi_{m}(\sqrt{\mathrm{H}}) u_{0}$ and $g_{m}=\phi_{m}(\sqrt{\mathrm{H}}) u_{1}$. Then we can write the solution

$$
u=u^{l}+u^{h}, \quad u^{l}=\sum_{m \leqslant-1} u_{m}, \quad u^{h}=\sum_{m \geqslant 0} u_{m} .
$$

Let $U(t)=e^{i t \sqrt{1+\mathrm{H}}}$, then we write

$$
u_{m}(t, z)=\frac{U(t)+U(-t)}{2} f_{m}+\frac{U(t)-U(-t)}{2 i \sqrt{1+\mathrm{H}}} g_{m} .
$$

Noting that

$$
U(t)=\sum_{j=1}^{N} \sum_{k \in \mathbb{Z}} U_{j, k}(t)=\sum_{j=1}^{N} U_{j}^{\text {low }}(t)+\sum_{j=1}^{N} \sum_{k \geqslant 0} U_{j, k}(t),
$$

we can write

$$
U(t) f=\sum_{j} \sum_{k \in \mathbb{Z}} \int_{0}^{\infty} e^{i t \sqrt{1+\lambda^{2}}} \phi\left(2^{-k} \lambda\right) Q_{j}(\lambda) \mathrm{d} E_{\sqrt{\mathrm{H}}}(\lambda) \widetilde{\phi}\left(2^{-k} \sqrt{\mathrm{H}}\right) f,
$$

where $\widetilde{\phi} \in C_{0}^{\infty}(\mathbb{R} \backslash\{0\})$ takes values in $[0,1]$ such that $\tilde{\phi} \phi=\phi$. In view of $f_{m}=$ $\phi\left(2^{-m} \sqrt{\mathrm{H}}\right) f$, then $\widetilde{\phi}\left(2^{-k} \sqrt{\mathrm{H}}\right) f_{m}$ vanishes if $|m-k| \geqslant 3$. Then we have

$$
U(t) f_{m}=\sum_{j} \sum_{|k-m| \leqslant 3} \int_{0}^{\infty} e^{i t \sqrt{1+\lambda^{2}}} \phi\left(2^{-k} \lambda\right) Q_{j}(\lambda) \mathrm{d} E_{\sqrt{\mathrm{H}}}(\lambda) f_{m} .
$$

By the squarefunction estimates (2.9) and Minkowski's inequality, we obtain for $q, r \geqslant$ 2

$$
\|u\|_{L^{q}\left(\mathbb{R} ; L^{r}(X)\right)} \lesssim\left\|u^{l}\right\|_{L^{q}\left(\mathbb{R} ; L^{r}(X)\right)}+\left(\sum_{m \geqslant 0}\left\|u_{m}\right\|_{L^{q}\left(\mathbb{R} ; L^{r}(X)\right)}^{2}\right)^{\frac{1}{2}} .
$$


To prove the homogeneous estimates in Theorem 1.1, that is $F=0$, we need

Proposition 4.2 Let $f_{m}=\phi_{m}(\sqrt{\mathrm{H}}) u_{0}$, we have for $m \geqslant 0$

$$
\left\|U(t) f_{m}\right\|_{L_{t}^{q} L_{z}^{r}(\mathbb{R} \times X)} \lesssim 2^{m s}\left\|f_{m}\right\|_{L^{2}(X)},
$$

where the $K-G$ admissible pair $(q, r) \in[2, \infty]^{2}$ and s satisfy (1.6) and (1.7).

Indeed, by using Propositions 3.3, 3.8, and the argument in Keel-Tao [29], we have for $2 / q \leqslant n(1 / 2-1 / r)$

$$
\left\|U_{j}^{\text {low }} u_{0}\right\|_{L^{q}\left(\mathbb{R} ; L^{r}(X)\right)} \leqslant C\left\|u_{0}\right\|_{L^{2}(X)}
$$

Without loss generality, we assume $u_{1}=0$. By using the Proposition 4.2, we have

$$
\begin{aligned}
& \left\|u^{l}\right\|_{L^{q}\left(\mathbb{R} ; L^{r}(X)\right)} \leqslant C \sum_{j=1}^{N}\left\|U_{j}^{\text {low }} u_{0}\right\|_{L^{q}\left(\mathbb{R} ; L^{r}(X)\right)} \leqslant C\left\|u_{0}\right\|_{L^{2}(X)}, \\
& \sum_{m \geqslant 0}\left\|u_{m}\right\|_{L^{q}\left(\mathbb{R} ; L^{r}(X)\right)}^{2} \leqslant C 2^{2 m s}\left\|f_{m}\right\|_{L^{2}(X)}^{2} \leqslant C\left\|u_{0}\right\|_{H^{s}(X)}^{2} .
\end{aligned}
$$

Therefore we prove the Strichartz estimate with $u_{1}=F=0$

$$
\|u\|_{L^{q}\left(\mathbb{R} ; L^{r}(X)\right)} \leqslant C\left\|u_{0}\right\|_{H^{s}(X)} .
$$

Now we prove this proposition. By using Propositions 3.2 and 3.9, we have the estimates (4.1) for $U_{j, k}(t)$, where $\alpha=(n+1+\theta) / 2, \sigma=(n-1+\theta) / 2$ and $h=2^{-k}$. Then it follows from Proposition 4.1 that

$$
\left\|U_{j, k}(t) f_{m}\right\|_{L_{t}^{q}\left(\mathbb{R}: L^{r}(X)\right)} \lesssim 2^{k\left[(n+\theta)\left(\frac{1}{2}-\frac{1}{r}\right)-\frac{1}{q}\right]}\left\|f_{m}\right\|_{L^{2}(X)}
$$

By (4.7), we obtain

$$
\left\|U(t) f_{m}\right\|_{L_{t}^{q}\left(\mathbb{R}: L^{r}(X)\right)} \lesssim 2^{m\left[(n+\theta)\left(\frac{1}{2}-\frac{1}{r}\right)-\frac{1}{q}\right]}\left\|f_{m}\right\|_{L^{2}(X)}=2^{m s}\left\|f_{m}\right\|_{L^{2}(X)}
$$

which proves (4.9).

\subsection{Inhomogeneous Strichartz Estimates}

In this subsection, we prove the inhomogeneous Strichartz estimates. Let $U(t)=$ $e^{i t \sqrt{1+\mathrm{H}}}: L^{2} \rightarrow L^{2}$. We have already proved that

$$
\left\|U(t) u_{0}\right\|_{L_{t}^{q} L_{z}^{r}} \lesssim\left\|u_{0}\right\|_{H^{s}}
$$


holds for all $(q, r, s)$ satisfying (1.6) and (1.7). For $s \in \mathbb{R}$ and $(q, r)$ satisfying (1.6) and (1.7), we define the operator $T_{S}$ by

$$
T_{s}: L_{z}^{2} \rightarrow L_{t}^{q} L_{z}^{r}, \quad f \mapsto(1+\mathrm{H})^{-\frac{s}{2}} e^{i t \sqrt{1+\mathrm{H}}} f
$$

Then we have by duality

$$
T_{1-s}^{*}: L_{t}^{\tilde{q}^{\prime}} L_{z}^{\tilde{r}^{\prime}} \rightarrow L^{2}, \quad F(\tau, z) \mapsto \int_{\mathbb{R}}(1+\mathrm{H})^{\frac{s-1}{2}} e^{-i \tau \sqrt{1+\mathrm{H}}} F(\tau) \mathrm{d} \tau,
$$

where $1-s=n\left(\frac{1}{2}-\frac{1}{\tilde{r}}\right)-\frac{1}{\tilde{q}}$. Therefore we obtain

$$
\left\|\int_{\mathbb{R}} U(t) U^{*}(\tau) \mathrm{H}^{-\frac{1}{2}} F(\tau) \mathrm{d} \tau\right\|_{L_{t}^{q} L_{z}^{r}}=\left\|T_{s} T_{1-s}^{*} F\right\|_{L_{t}^{q} L_{z}^{r}} \lesssim\|F\|_{L_{t}^{\tilde{q}^{\prime}} L_{z}^{\tilde{r}^{\prime}}}
$$

Since $s=n\left(\frac{1}{2}-\frac{1}{r}\right)-\frac{1}{q}$ and $1-s=n\left(\frac{1}{2}-\frac{1}{\tilde{r}}\right)-\frac{1}{\tilde{q}}$, thus $(q, r),(\tilde{q}, \tilde{r})$ satisfy (1.7). By the Christ-Kiselev lemma [13], we thus obtain for $q>\tilde{q}^{\prime}$,

$$
\left\|\int_{\tau<t} \frac{\sin (t-\tau) \sqrt{1+\mathrm{H}}}{\sqrt{1+\mathrm{H}}} F(\tau) \mathrm{d} \tau\right\|_{L_{t}^{q} L_{z}^{r}} \lesssim\|F\|_{L_{t}^{\tilde{q}^{\prime}} L_{z}^{\tilde{r}^{\prime}}}
$$

Notice that for all $(q, r),(\tilde{q}, \tilde{r})$ satisfy (1.6) and (1.7), we must have $q>\tilde{q}^{\prime}$. Therefore we have proved all inhomogeneous Strichartz estimates including $q=2$.

\section{Well-posedness and Small Nonlinear Scattering}

In this section, we prove Theorems 1.3 and 1.5 . We prove the results by a contraction mapping argument. The key point is the application of Strichartz estimates.

\subsection{Proof of Theorem 1.3}

Let $q_{0}=(n+1)(p-1) / 2, q_{1}=2(n+1) /(n-1)$ and $\alpha=s_{c}-\frac{1}{2}$. For any small constant $\epsilon>0$ such that

$$
\begin{gathered}
Y:=\left\{u: u \in C_{t}\left(H^{s_{c}}\right) \cap L^{q_{0}}\left([0, T] ; L^{q_{0}}(X)\right) \cap L^{q_{1}}\left([0, T] ; H_{q_{1}}^{\alpha}(X)\right),\right. \\
\left.\|u\|_{L^{q_{0}}\left([0, T] ; L^{q_{0}}(X)\right)}+\|u\|_{L^{q_{1}}\left([0, T] ; H_{q_{1}}^{\alpha}(X)\right)} \leqslant C \epsilon\right\} .
\end{gathered}
$$


Consider the solution map $\Phi$ defined by

$$
\begin{aligned}
\Phi(u)= & \cos (t \sqrt{1+\mathrm{H}}) u_{0}(z)+\frac{\sin (t \sqrt{1+\mathrm{H}})}{\sqrt{1+\mathrm{H}}} u_{1}(z) \\
& +\int_{0}^{t} \frac{\sin ((t-s) \sqrt{1+\mathrm{H}})}{\sqrt{1+\mathrm{H}}} F(u(s, z)) \mathrm{d} s \\
= & : u_{\mathrm{hom}}+u_{\mathrm{inh}},
\end{aligned}
$$

where $F(u)= \pm|u|^{p-1} u$. We claim the map $\Phi: Y \rightarrow Y$ is contracting. We first note that the Sobolev embedding $L_{t}^{q_{0}} H_{r_{0}}^{\alpha} \hookrightarrow L_{t, z}^{q_{0}}$ where $r_{0}=\left(\frac{\alpha}{n}+\frac{1}{q_{0}}\right)^{-1}$. Since $p \geqslant 1+4 /(n-1)$, thus $s_{c} \geqslant 1 / 2$. On the other hand, it is easy to check that the pairs $\left(q_{0}, r_{0}\right),\left(q_{1}, q_{1}\right)$ satisfy (1.6) and (1.7) with $s=1 / 2$ and $\theta=0$. By Theorem 1.1, we obtain

$$
\left\|u_{\mathrm{hom}}\right\|_{C_{t}\left(H^{s_{c}}\right) \cap L^{q_{0}}\left(\mathbb{R} ; L^{q_{0}}(X)\right) \cap L^{q_{1}}\left(\mathbb{R} ; H_{q_{1}}^{\alpha}(X)\right)} \leqslant C\left(\left\|u_{0}\right\|_{H^{s_{c}}}+\left\|u_{1}\right\|_{H^{s_{c}-1}}\right) .
$$

Hence we must have

$$
\left\|u_{\mathrm{hom}}\right\|_{L^{q_{0}}\left([0, T] ; L^{q_{0}}(X)\right) \cap L^{q_{1}}\left([0, T] ; H_{q_{1}}^{\alpha}(X)\right)} \leqslant \frac{1}{2} C \epsilon
$$

for $T=\infty$ if the initial data have small norm $\epsilon(p)$, or, if not, this inequality will be satisfied for some $T>0$ by the dominated convergence theorem. Applying Theorem 1.1 with $\tilde{q}^{\prime}=\tilde{r}^{\prime}=\frac{2(n+1)}{n+3}$, one has

$$
\left\|u_{\mathrm{inh}}\right\|_{C_{t}\left(H^{s_{c}}\right) \cap L^{q_{0}}\left([0, T] ; L^{q_{0}}(X)\right) \cap L^{q_{1}}\left([0, T] ; H_{q_{1}}^{\alpha}(X)\right) \leqslant C\|F(u)\|_{L_{t}^{\tilde{q}^{\prime}}} H_{\tilde{r}^{\prime}}^{\alpha}} .
$$

Note $p \in\left[1+\frac{4}{n-1}, 1+\frac{4}{n-2}\right]$, we have $0 \leqslant \alpha \leqslant 1$. By using the fraction Liebniz rule for Sobolev spaces in Proposition 2.3, we have

$$
\|F(u)\|_{L_{t}^{\tilde{q}^{\prime}} H_{\tilde{r}^{\prime}}^{\alpha}} \leqslant C\|u\|_{L_{t, z}^{q_{0}}}^{p-1}\|u\|_{L_{t}^{q_{1}} H_{q_{1}}^{\alpha}} \leqslant C^{2}(C \epsilon)^{p-1} \epsilon \leqslant \frac{C \epsilon}{2} .
$$

A similar argument as above leads to

$$
\begin{aligned}
& \left\|\Phi\left(u_{1}\right)-\Phi\left(u_{2}\right)\right\|_{L^{q_{1}}\left([0, T] ; H_{q_{1}}^{\alpha}(X)\right) \cap L^{q_{0}}\left([0, T] ; L^{q_{0}}(X)\right)} \\
& \quad \leqslant C\left\|F\left(u_{1}\right)-F\left(u_{2}\right)\right\|_{L_{t}^{q^{\prime}}} H_{\tilde{r}^{\prime}}^{\alpha} \\
& \quad \leqslant C^{2}(C \epsilon)^{p-1}\left\|u_{1}-u_{2}\right\|_{L^{q_{1}}\left([0, T] ; H_{q_{1}}^{\alpha}(X)\right) \cap L^{q_{0}}\left([0, T] ; L^{q_{0}}(X)\right)} \\
& \quad \leqslant \frac{1}{2}\left\|u_{1}-u_{2}\right\|_{L^{q_{1}}\left([0, T] ; H_{q_{1}}^{\alpha}(X)\right) \cap L^{q_{0}}\left([0, T] ; L^{q_{0}}(X)\right) .}
\end{aligned}
$$

Therefore the solution map $\Phi$ is a contraction map on $Y$ under the metric $d\left(u_{1}, u_{2}\right)=$ $\left\|u_{1}-u_{2}\right\|_{L^{q_{1}}\left([0, T] ; H_{q_{1}}^{\alpha}(X)\right) \cap L^{q_{0}}\left([0, T] ; L^{q_{0}}(X)\right)}$. The standard contraction argument proves 
the first part of Theorem 1.3. Note that the above argument needs the condition $p \in$ $\left[1+\frac{4}{n-1}, 1+\frac{4}{n-2}\right]$ in (5.2). If $\left(u_{0}, u_{1}\right) \in H^{1}(X) \times L^{2}(X)$, we extend the local wellposedness for $p \in\left[p_{\text {conf }}, 1+\frac{4}{n-2}\right]$. By energy conservation law, we obtain the global existence for large data and finish the final part of Theorem 1.3.

\subsection{Proof of Theorem 1.5}

For a constant $C$ we define

$$
\widetilde{Y}:=\left\{u:\|u\|_{C_{t}\left([0, T] ; H^{1+\delta}\right) \cap L^{2}\left([0, T] ; L^{\infty}(X)\right)} \leqslant 2 C\right\} .
$$

Consider the solution map $\Phi$ defined by

$$
\begin{aligned}
\Phi(u)= & \cos (t \sqrt{1+\mathrm{H}}) u_{0}(z)+\frac{\sin (t \sqrt{1+\mathrm{H}})}{\sqrt{1+\mathrm{H}}} u_{1}(z) \\
& +\int_{0}^{t} \frac{\sin ((t-s) \sqrt{1+\mathrm{H}})}{\sqrt{1+\mathrm{H}}} F(u(s, z)) \mathrm{d} s \\
= & : u_{\mathrm{hom}}+u_{\mathrm{inh}},
\end{aligned}
$$

where $F(u)$ is replaced by $F(u)=u D u+|u|^{2} u$. By Theorem 1.1 with $0<\theta=$ $2 \delta \ll 1$, we obtain

$$
\left\|u_{\mathrm{hom}}\right\|_{C_{t}\left([0, T] ; H^{1+\delta}\right) \cap L^{2}\left([0, T] ; L^{\infty}(X)\right)} \leqslant C\left(\left\|u_{0}\right\|_{H^{1+\delta}}+\left\|u_{1}\right\|_{H^{\delta}}\right) .
$$

Furthermore one has by Theorem 1.1 and choosing small $T$

$$
\begin{aligned}
& \left\|u_{\mathrm{inh}}\right\|_{C_{t}\left([0, T] ; H^{1+\delta}\right) \cap L^{2}\left([0, T] ; L^{\infty}(X)\right)} \\
& \leqslant C\|u D u\| \frac{1}{L_{t}^{1-\delta}} L^{2}(X) \\
& \quad+C\left\|u^{3}\right\| \frac{1}{L_{t}^{1-\delta}} L^{2}(X) \\
& \leqslant C T^{\frac{1}{2}-\delta}\|u\|_{L^{2}\left([0, T] ; L^{\infty}(X)\right)}\|D u\|_{L_{t}^{\infty}\left([0, T] ; L^{2}(X)\right)} \\
& \quad+C T^{(1-\delta) / 3}\|u\|_{L_{t}^{\infty} L^{6}(X)}^{3} \leqslant 2 C .
\end{aligned}
$$

By choosing $T$ small enough, we have

$$
\begin{aligned}
& \left\|\Phi\left(u_{1}\right)-\Phi\left(u_{2}\right)\right\|_{C_{t}\left([0, T] ; H^{1+\delta}\right) \cap L^{2}\left([0, T] ; L^{\infty}(X)\right)} \\
& \leqslant C T^{\frac{1}{4}}\left(\left\|u_{1}-u_{2}\right\|_{L^{2}\left([0, T] ; L^{\infty}(X)\right)}+\left\|D\left(u_{1}-u_{2}\right)\right\|_{L_{t}^{\infty}\left([0, T] ; L^{2}(X)\right)}\right. \\
& \left.\quad+\left\|u_{1}-u_{2}\right\|_{L_{t}^{\infty} L^{6}(X)}\right) \\
& \leqslant \frac{1}{2}\left\|u_{1}-u_{2}\right\|_{C_{t}\left([0, T] ; H^{1+\delta}\right) \cap L^{2}\left([0, T] ; L^{\infty}(X)\right)} .
\end{aligned}
$$

The standard contraction argument on $\widetilde{Y}$ completes the proof of Theorem 1.5. 
Acknowledgements The authors would like to thank Andrew Hassell, Changxing Miao, and Andras Vasy for their helpful discussions and encouragement. This work was supported by the National Natural Science Foundation of China (11771041,11401024,11831004), China Scholarship Council, and the European Research Council, ERC-2012-ADG, Project Number 320845: Semi Classical Analysis of Partial Differential Equations.

Open Access This article is distributed under the terms of the Creative Commons Attribution 4.0 International License (http://creativecommons.org/licenses/by/4.0/), which permits unrestricted use, distribution, and reproduction in any medium, provided you give appropriate credit to the original author(s) and the source, provide a link to the Creative Commons license, and indicate if changes were made.

\section{References}

1. Alexopoulos, G.: Spectral multipliers for Markov chains. JMS Jpn. 56, 833-852 (2004)

2. Anker, J.P., Pierfelice, V.: Wave and Klein-Gordon equations on hyperbolic spaces. Anal. PDE 7, 953-995 (2014)

3. Brenner, P.: On space-time means and everywhere defined scattering operators for nonlinear KleinGordon equations. Math. Z. 186, 383-391 (1984)

4. Baskin, D.: A parametrix for the forward fundamental solution of the Klein-Gordon equation on asymptotically de Sitter spaces. J. Funct. Anal. 259, 1673-1719 (2010)

5. Baskin, D.: A Strichartz estimate for de Sitter space. Proc. Centre Math. Appl. ANU 44, 97-104 (2010)

6. Baskin, D.: Strichartz estimates on asymptotically de Sitter spaces. Ann. Henri Poincaré 14, 221-252 (2013)

7. Bouclet, J.M., Mizutani, H.: Global in time Strichartz inequalities on asymptotically flat manifolds with temperate trapping. arXiv:1602.06287v1

8. Blair, M.D., Smith, H.F., Sogge, C.D.: Strichartz estimates for the wave equation on manifold with boundary. Ann. Inst. H. Poincaré Anal. Non Linéaire 26, 1817-1829 (2009)

9. Blair, M.D., Ford, G.A., Marzuola, J.L.: Strichartz estimates for the wave equation on flat cones. Int. Math. Res. Not. 3, 562-591 (2013)

10. Burq, N.: Global Strichartz estimates for nontrapping geometries: about an article by H. Smith and C. D. Sogge. Commun. PDE 28, 1675-1683 (2003)

11. Burq, N., Lebeau, G., Planchon, F.: Global existence for energy critical waves in 3-D domains. J. Am. Math. Soc. 21, 831-845 (2008)

12. Cheng, S.Y., Li, P., Yau, S.T.: On the upper estimate of a complete Riemannian manifold. Am. J. Math. 103, 1021-1063 (1981)

13. Christ, M., Kiselev, A.: Maxiamal functions associated to filtrations. J. Funct. Anal. 179, 409-425 (2001)

14. Coulhon, T., Russ, E., Tardivel-Nachef, V.: Sobolev algebras on Lie groups and Riemannian manifolds. Am. J. Math. 123, 283-342 (2001)

15. Hintz, P.: Global well-posedness of quasilinear wave equations on asymptotically de Sitter spaces. Ann. l'Inst. Fourier 66, 1285-1408 (2016)

16. Hintz, P., Vasy, A.: Semilinear wave equations on asymptotically de Sitter. Kerr-de Sitter and Minkowski spacetimes. Anal. PDE 8, 1807-1890 (2015)

17. Hintz, P., Vasy, A.: Global analysis of quasilinear wave equations on asymptotically Kerr-de Sitter spaces. Int. Math. Res. Notices (2015). https://doi.org/10.1093/imrn/rnv311

18. Ginibre, J., Velo, G.: Time decay of finite energy solutions of the nonlinear Klein-Gordon and Schrödinger equations. Ann. Inst. Henri Poincare 43, 339-442 (1985)

19. Guillarmou, C., Hassell, A., Sikora, A.: Resolvent at low energy III: the spectral measure. Trans. Am. Math. Soc. 365, 6103-6148 (2013)

20. Guillarmou, C., Hassell, A., Sikora, A.: Restriction and spectral multiplier theorems on asymptotically conic manifolds. Anal. PDE 6, 893-950 (2013)

21. Hassell, A., Tao, T., Wunsch, J.: A Strichartz inequality for the Schrödinger equation on non-trapping asymptotically conic manifolds. Commun. PDE 30, 157-205 (2005)

22. Hassell, A., Tao, T., Wunsch, J.: Sharp Strichartz estimates on non-trapping asymptotically conic manifolds. Am. J. Math. 128, 963-1024 (2006) 
23. Hassell, A., Wunsch, J.: The semiclassical resolvent and propagator for non-trapping scattering metrics. Adv. Math. 217, 586-682 (2008)

24. Hassell, A., Wunsch, J.: The Schrödinger propagator for scattering metrics. Ann. Math. 162, 487-523 (2005)

25. Hassell, A., Vasy, A.: The spectral projections and resolvent for scattering metrics. J. Anal. Math. 79, 241-298 (1999)

26. Hassell, A., Zhang, J.: Global-in-time Strichartz estimates on non-trapping asymptotically conic manifolds. Anal. PDE 9, 151-192 (2016)

27. Ibrahim, S., Masmoudi, N., Nakanishi, K.: Scattering threshold for the focusing nonlinear KleinGordon equation. Anal. PDE 4, 405-460 (2011)

28. Kapitanski, L.V.: Norm estimates in Besov and Lizorkin-Treibel spaces for the solution of second order linear hyperbolic equations. J. Sov. Math. 56, 2348-2389 (1991)

29. Keel, M., Tao, T.: Endpoint Strichartz estimates. Am. J. Math. 120, 955-980 (1998)

30. Koch, H., Tataru, D., Zworski, M.: Semiclassical $L^{p}$ estimates. Ann. Henri PoincarPoincaré 8, 885-916 (2007)

31. Lindblad, H.: A sharp counterexample to the local existence of low-regularity solutions to nonlinear wave equations. Duke Math. J. 72, 503-539 (1993)

32. Lindblad, H., Sogge, C.D.: On existence and scattering with minimal regularity for semi-linear wave equations. J. Funct. Anal. 130, 357-426 (1995)

33. Melrose, R.B.: The Atiyah-Patodi-Singer Index. Theorem Research Notes in Mathematics, vol. 4. Peters, Wellesley (1993)

34. Melrose, R.B.: Spectral and scattering theory for the Laplacian on asymptotically Euclidian spaces. In: Spectral and Scattering Theory (Sanda, 1992). Lecture Notes in Pure and Appl. Math., Vol. 161, pp. 85-130. Dekker, New York (1994)

35. Metcalfe, J.: Global Strichartz estimates for solutions to the wave equation exterior to a convex obstacle. Trans. Am. Math. Soc. 356, 4839-4855 (2004)

36. Nakanishi, K., Schlag, W.: Invariant Manifolds and Dispersive Hamiltonian Evolution Equations. European Mathematical Society, Zurich (2011)

37. Ponce, G., Sideris, T.: Local regularity of nonlinear wave equations in three space dimensions. Commun. PDE 18, 169-177 (1993)

38. Strauss, W.A.: Nonlinear scattering theory at low energy. J. Funct. Anal. 41, 110-133 (1981)

39. Smith, H.F.: A parametrix construction for wave equation with $C^{1,1}$ coefficients. Ann. Inst. Fourier 48, 797-836 (1998)

40. Smith, H.F., Sogge, C.D.: Global Strichartz estimates for nontrapping perturbations of the Laplacian. Commun. PDE 25, 2171-2183 (2000)

41. Stein, E.M.: Singular Integrals and Differentiability Properties of Functions. Princeton University Press, Princeton (1970)

42. Sogge, C.D.: Lectures on Nonlinear Wave Equations. International Press, Cambridge, MA (1995)

43. Tataru, D.: Strichartz estimates for second order hyperbolic operators with nonsmooth coefficients III. J. Am. Math. Soc. 15, 419-442 (2002)

44. Taylor, M.E.: Tools for PDE. Mathematical Surveys and Monographs, vol. 81. American Mathematical Society, Providence, RI (2000)

45. Vasy, A.: Microlocal Analysis of Asymptotically Hyperbolic and Kerr-de Sitter Spaces (with an Appendix by Semyon Dyatlov). Inventiones Mathematicae, pp. 1-133 (2013)

46. Zworski, M.: Semiclassical Analysis. Graduate Studies in Mathematics, vol. 138. AMS, New York (2012)

47. Zhang, J.: Strichartz estimates and nonlinear wave equation on nontrapping asymptotically conic manifolds. Adv. Math. 271, 91-111 (2015) 